\title{
Heteroscedastic semiparametric transformation models: estimation and testing for validity
}

\author{
Natalie NeumeYer ${ }^{*}$ Hohsuk NoH $^{\dagger}$ Ingrid VAN KeILEGOM ${ }^{\ddagger}$
}

August 25, 2015

\begin{abstract}
In this paper we consider a heteroscedastic transformation model of the form $\Lambda_{\vartheta}(Y)=m(X)+\sigma(X) \varepsilon$, where $\Lambda_{\vartheta}$ belongs to a parametric family of monotone transformations, $m(\cdot)$ and $\sigma(\cdot)$ are unknown but smooth functions, $\varepsilon$ is independent of the $d$-dimensional vector of covariates $X, E(\varepsilon)=0$ and $\operatorname{Var}(\varepsilon)=1$. In this model, we first consider the estimation of the unknown components of the model, namely $\vartheta, m(\cdot), \sigma(\cdot)$ and the distribution of $\varepsilon$, and we show the asymptotic normality of the proposed estimators. Second, we propose tests for the validity of the model, and establish the limiting distribution of the test statistics under the null hypothesis. A bootstrap procedure is proposed to approximate the critical values of the tests. Finally, we carry out a simulation study to verify the small sample behavior of the proposed estimators and tests and illustrate our method with a real dataset.
\end{abstract}

Key words: bootstrap, empirical distribution function, empirical independence process, local polynomial estimator, location-scale model, model specification, nonparametric regression, profile likelihood estimator

AMS 2010 classification: 62G05, 62G08, 62G09, 62G10, 62G30

*Department of Mathematics, University of Hamburg, Bundesstrasse 55, 20146 Hamburg, Germany, E-mail: neumeyer@math.uni-hamburg.de

†Department of Statistics, Sookmyung Women's University, 100 Cheongpa-ro 47-gil, Yongsan-gu, Seoul, South Korea 140-742, E-mail: word5810@gmail.com

¥Institute of Statistics, Université catholique de Louvain, Voie du Roman Pays 20, 1348 Louvain-laNeuve, Belgium, E-mail: ingrid.vankeilegom@uclouvain.be 


\section{Introduction}

Assume we observe independent copies of a random vector $(X, Y)$, where $X$ represents a $d$-dimensional covariate and $Y$ is a univariate response. One possibility is to analyze these data by fitting a non- or semiparametric regression model, i. e.

$$
Y=m(X)+\varepsilon, \text { where } E[\varepsilon \mid X]=0 .
$$

Doing so, often the conditional error distribution, given the covariate, still depends on $X$, which means that the dependency of the response $Y$ on the covariate $X$ goes beyond the first moment. If only the second moment is dependent on $X$ one can fit a nonparametric location-scale model of the form

$$
Y=m(X)+\sigma(X) \varepsilon, \text { where } \varepsilon \perp X \text { with } E[\varepsilon]=0, \operatorname{Var}(\varepsilon)=1 .
$$

Here and throughout the paper $Z \perp X$ means that $Z$ and $X$ are stochastically independent. Such nonparametric location-scale models have been widely used, see e.g. Akritas and Van Keilegom (2001), Dette, von Lieres und Wilkau and Sperlich (2005) or Hušková and Meintanis (2010), among many others. Note that the conditional normal distribution is always a special case because from $Y \mid X=x \sim N\left(m(x), \sigma^{2}(x)\right)$ it follows that $\varepsilon \sim N(0,1)$ does not depend on $X$. The general location-scale model (1.2) has several advantages over the unstructured model (1.1). First, the asymptotic analysis of statistical procedures often simplifies a lot. Further, the model allows to estimate the error distribution with a parametric $\sqrt{n}$-rate, see Akritas and Van Keilegom (2001). Therefore the estimation of the conditional distribution of $Y$ given $X$ is much more efficient. Goodness-of-fit as well as other specification tests have been developed that specifically use the location-scale structure, see Section 2.4 in the recent review by González-Manteiga and Crujeiras (2013). When data $\left(X, Y_{1}, Y_{2}\right)$ have been observed and one's interest lies in the dependence between $Y_{1}$ and $Y_{2}$, given $X$, under the location-scale structure the conditional copula of $\left(Y_{1}, Y_{2}\right)$, given $X$, can not only be estimated with $\sqrt{n}$-rate, but also as precisely as if the errors would be known, see Gijbels, Omelka and Veraverbeke (2015).

The construction of valid resampling procedures is essential for most hypothesis tests in nonparametric regression. It is known that in heteroscedastic regression models simple residual bootstrap methods generally do not lead to valid procedures. Thus mostly wild bootstrap is used, see Härdle and Mammen (1993) and Stute, González Manteiga and Presedo Quindimil (1998). However, Zhu, Fujikoshi and Naito (2001) show that wild bootstrap may fail if the conditional 4th moment of the error distribution depends on the 
covariate, while for the procedure considered there it works in the location-scale context. There are other cases where wild bootstrap even fails in the location-scale model (1.2), see e.g. Neumeyer and Sperlich (2006). A (smooth or not smooth) heteroscedastic residual bootstrap often can be an alternative, see Neumeyer (2009a), and explicitly makes use of the location-scale structure.

Before application of model (1.2) a specification test should be conducted, i. e. a test for independence of $\varepsilon$ and $X$. Such tests have been suggested by Einmahl and Van Keilegom (2008), Neumeyer (2009b), and Hlávka, Hušková and Meintanis (2011). However, if those tests reject the null hypothesis a remedy might be to transform the response $Y$ by a suitable transformation $\Lambda$ before fitting the location-scale model to the data $(X, Y)$.

It is very common in practice to transform the response variable before fitting a regression model to the data. The aim of the transformation is to reduce skewness or heteroscedasticity, or to induce normality. Often the transformation is chosen from a parametric class such as the famous class of Box-Cox power transformations introduced by Box and Cox (1964). Generalizations of this class were suggested by Bickel and Doksum (1981) and Yeo and Johnson (2000), among others. The parameter of the transformation in the class can be chosen data dependently by a profile likelihood approach, for instance. There is a huge literature on parametric transformation models and we refer to the monograph by Carroll and Ruppert (1988); see also the references in Fan and Fine (2013). Nonparametric estimation of the transformation in the context of parametric regression models has been considered by Horowitz (1996) and Zhou, Lin and Johnson (2009), among others. Horowitz (2009) reviews estimation in transformation models with parametric regression in the cases where either the transformation or the error distribution or both are modeled nonparametrically. Linton, Sperlich and Van Keilegom (2008) consider a parametric class of transformations, while the error distribution is estimated nonparametrically and the regression function is assumed to be additive. The aim of the transformation is to induce independence of the covariate and the error. Asymptotic normality of a profile likelihood estimator for the transformation parameter is proved. Heuchenne, Samb and Van Keilegom (2015) consider a residual based empirical distribution function in the same model in order to estimate the error distribution. Recently, Colling and Van Keilegom (2015) considered goodness-of-fit tests for the regression function in a semiparametric transformation model, in which the transformation parameter is estimated by means of the profile likelihood estimator of Linton et al. (2008).

The aim of our paper is twofold. On one hand we generalize the results of Linton et al. 
(2008) by allowing heteroscedasticity. To this end in a parametric class of transformations we seek the one that leads to a nonparametric location-scale model of the form

$$
\Lambda(Y)=m(X)+\sigma(X) \varepsilon, \text { where } \varepsilon \perp X \text { with } E[\varepsilon]=0, \operatorname{Var}(\varepsilon)=1,
$$

where $\Lambda$ denotes the transformation. The regression function $m$ and variance function $\sigma^{2}$ are modeled fully nonparametrically, but analogous results can be obtained for semiparametric modeling. We estimate the transformation parameter by a profile-likelihood approach and prove asymptotic normality of the estimator. We investigate the performance of the estimator in a simulation study. Note that in the context of parametric regression, Zhou et al. (2009) and Khan, Shin and Tamer (2011) considered heteroscedastic transformation models.

On the other hand for the first time in the literature a test for model validity in the context of transformation models with parametric class of transformations and non- (or semi-)parametric regression function is proposed. $\mathrm{Mu}$ and $\mathrm{He}$ (2007) consider estimation procedures in a transformation model with linear quantile regression function and also suggest a test for model validity. In the general heteroscedastic case we suggest tests for the hypothesis of existence of some transformation $\Lambda$ in the considered parametric class such that the data fulfill model (1.3). The results can readily be modified to test whether such a model can hold with $\sigma \equiv 1$, i. e. a homoscedastic transformation model. Our test statistics are based on the difference between the estimated joint distribution of covariables and errors and the product of the marginal distributions. A similar approach was used to test for validity of a location-scale model (without transformation) by Einmahl and Van Keilegom (2008). However, the estimation of the unknown transformation vastly complicates the theoretical derivations. We show weak convergence of the estimated empirical process to a centered Gaussian process under the null hypothesis of model validity. As a by-product we obtain an expansion for the residual-based empirical distribution function that generalizes results by Heuchenne et al. (2015). Moreover, we discuss consistency of the proposed tests and demonstrate the finite sample properties of a bootstrap version of Kolmogorov-Smirnov and Cramér von Mises tests in a simulation study.

The rest of the paper is organized as follows. In Section 2 we define the profile likelihood estimator for the transformation parameter and show asymptotic normality. We further discuss estimation of the regression and variance function by local polynomial estimators, and the estimation of the error distribution. In Section 3 we consider the problem of testing for existence of a transformation in the considered class that leads to 
a location-scale model. We derive an expansion for the estimator of the joint distribution of covariates and errors. Under the null hypothesis we show weak convergence of the process given by the difference of the estimated joint distribution and the product of the marginals. Consistency of the testing procedures and modifications for the homoscedastic model are discussed. Additionally, we describe bootstrap versions of the hypothesis tests. In Section 4, we also present simulations to demonstrate finite sample properties of the profile likelihood estimator for the transformation parameter as well as the hypothesis tests. Further, we illustrate our method with a real dataset. All regularity conditions and some of the proofs are collected in Appendices A and B. The other proofs are in a supplementary document.

\section{Estimation of the model}

Let $L=\left\{\Lambda_{\vartheta} \mid \vartheta \in \Theta\right\}$ be some parametric class of differentiable and strictly increasing transformations, and let $\Theta$ be some nonempty subset of $\mathbb{R}^{k}$. In this section we assume that there exists some unique $\vartheta_{0} \in \Theta$ such that

$$
\frac{\Lambda_{\vartheta_{0}}(Y)-E\left[\Lambda_{\vartheta_{0}}(Y) \mid X\right]}{\left(\operatorname{Var}\left(\Lambda_{\vartheta_{0}}(Y) \mid X\right)\right)^{1 / 2}} \perp X
$$

Then the covariate and transformed response can be modeled by a nonparametric locationscale model, i. e.

$$
\Lambda_{\vartheta_{0}}(Y)=m(X)+\sigma(X) \varepsilon, \quad \varepsilon \perp X,
$$

where $m(x)=E\left[\Lambda_{\vartheta_{0}}(Y) \mid X=x\right]$ and $\sigma^{2}(x)=\operatorname{Var}\left(\Lambda_{\vartheta_{0}}(Y) \mid X=x\right)$.

\subsection{Estimation of the transformation parameter}

To estimate the transformation parameter $\vartheta_{0}$ we will use a profile likelihood approach. This type of approach has been proposed by Linton et al. (2008) in the context of homoscedastic transformation models, and has been further used by Heuchenne et al. (2015) and Colling and Van Keilegom (2015) in the context of the estimation of the error distribution and the development of goodness-of-fit tests for the regression function, respectively. We will extend their method to the current setup with heteroscedastic errors.

For $\vartheta \in \Theta$, let $m_{\vartheta}(x)=E\left[\Lambda_{\vartheta}(Y) \mid X=x\right], \sigma_{\vartheta}^{2}(x)=\operatorname{Var}\left[\Lambda_{\vartheta}(Y) \mid X=x\right]$, and

$$
\varepsilon(\vartheta)=\frac{\Lambda_{\vartheta}(Y)-m_{\vartheta}(X)}{\sigma_{\vartheta}(X)} .
$$


Also, let $F_{\varepsilon(\vartheta)}(y)=P(\varepsilon(\vartheta) \leq y)$ denote the marginal distribution function of the errors and let $f_{\varepsilon(\vartheta)}(y)$ be the corresponding probability density function. We use the abbreviated notations $\Lambda=\Lambda_{\vartheta_{0}}, \varepsilon=\varepsilon\left(\vartheta_{0}\right), m=m_{\vartheta_{0}}, \sigma^{2}=\sigma_{\vartheta_{0}}^{2}, F_{\varepsilon}=F_{\varepsilon\left(\vartheta_{0}\right)}$ and $f_{\varepsilon}=f_{\varepsilon\left(\vartheta_{0}\right)}$.

Then, the conditional distribution $F_{Y \mid X}(\cdot \mid x)$ of $Y$ given $X=x$ can be written as

$$
F_{Y \mid X}(y \mid x)=F_{\varepsilon}\left(\frac{\Lambda(y)-m(x)}{\sigma(x)}\right)
$$

and hence the conditional density $f_{Y \mid X}(\cdot \mid x)$ of $Y$ given $X=x$ equals

$$
f_{Y \mid X}(y \mid x)=f_{\varepsilon}\left(\frac{\Lambda(y)-m(x)}{\sigma(x)}\right) \frac{\Lambda^{\prime}(y)}{\sigma(x)} .
$$

Assume we have independent observations $\left(X_{i}, Y_{i}\right), i=1, \ldots, n$, from the same distribution as $(X, Y)$ and let $\varepsilon_{i}=\varepsilon_{i}\left(\vartheta_{0}\right), i=1, \ldots, n$. Then, for an arbitrary value $\vartheta \in \Theta$, the log-likelihood can be written as

$$
L_{\vartheta}=\sum_{i=1}^{n}\left\{\log f_{\varepsilon(\vartheta)}\left(\frac{\Lambda_{\vartheta}\left(Y_{i}\right)-m_{\vartheta}\left(X_{i}\right)}{\sigma_{\vartheta}\left(X_{i}\right)}\right)+\log \Lambda_{\vartheta}^{\prime}\left(Y_{i}\right)-\log \sigma_{\vartheta}\left(X_{i}\right)\right\} .
$$

In order to maximize this log-likelihood with respect to $\vartheta$, we first need to replace the unknown functions $f_{\varepsilon(\vartheta)}, m_{\vartheta}$ and $\sigma_{\vartheta}$ by suitable estimators. For each $\vartheta \in \Theta$ we estimate $m_{\vartheta}(x)$ by a local polynomial estimator based on $\left(X_{i}, \Lambda_{\vartheta}\left(Y_{i}\right)\right), i=1, \ldots, n$. To this end denote the components of $X_{i}$ by $\left(X_{i 1}, \ldots, X_{i d}\right)(i=1, \ldots, n)$ and let $x=\left(x_{1}, \ldots, x_{d}\right)$. Let $\hat{m}_{\vartheta}(x)=\hat{\beta}_{0}$, where $\hat{\beta}_{0}$ is the first component of the vector $\hat{\beta}$, which is the solution of the local minimization problem

$$
\min _{\beta} \sum_{i=1}^{n}\left\{\Lambda_{\vartheta}\left(Y_{i}\right)-P_{i}(\beta, x, p)\right\}^{2} K_{h}\left(X_{i}-x\right) .
$$

Here, $P_{i}(\beta, x, p)$ is a polynomial of order $p$ built up with all $0 \leq k \leq p$ products of factors of the form $X_{i j}-x_{j}(j=1, \ldots, d)$. The vector $\beta$ is the vector consisting of all coefficients of this polynomial. Here, for $u=\left(u_{1}, \ldots, u_{d}\right) \in \mathbb{R}^{d}, K(u)=\prod_{j=1}^{d} k\left(u_{j}\right)$ is a $d$-dimensional product kernel, $k$ is a univariate kernel function, $h=\left(h_{1}, \ldots, h_{d}\right)$ is a $d$-dimensional bandwidth vector converging to zero when $n$ tends to infinity, and $K_{h}(u)=\prod_{j=1}^{d} k\left(u_{j} / h_{j}\right) / h_{j}$.

Analogously, for each $\vartheta \in \Theta$ let $\hat{s}_{\vartheta}$ denote a local polynomial estimator based on $\left(X_{i}, \Lambda_{\vartheta}\left(Y_{i}\right)^{2}\right), i=1, \ldots, n$, and define the variance function estimator as $\hat{\sigma}_{\vartheta}^{2}=\hat{s}_{\vartheta}-\hat{m}_{\vartheta}^{2}$. Note that this estimator has similar properties as a local polynomial estimator based on $\left(X_{i},\left(\Lambda_{\vartheta}\left(Y_{i}\right)-\hat{m}_{\vartheta}\left(X_{i}\right)\right)^{2}\right), i=1, \ldots, n$. 
Finally, let $\hat{\varepsilon}_{i}(\vartheta)=\left(\Lambda_{\vartheta}\left(Y_{i}\right)-\hat{m}_{\vartheta}\left(X_{i}\right)\right) / \hat{\sigma}_{\vartheta}\left(X_{i}\right)$ and define

$$
\hat{f}_{\hat{\varepsilon}(\vartheta)}(y)=\frac{1}{n} \sum_{i=1}^{n} \ell_{g}\left(\hat{\varepsilon}_{i}(\vartheta)-y\right)
$$

where $\ell$ and $g$ are a kernel function and a bandwidth sequence, possibly different from the kernel $k$ and the bandwidth $h$ that were used to estimate the regression and variance function.

Next, we plug in the estimators $\hat{m}_{\vartheta}, \hat{\sigma}_{\vartheta}$ and $\hat{f}_{\hat{\varepsilon}(\vartheta)}$ into the log-likelihood given in $(2.2)$ and obtain the following profile likelihood estimator of $\vartheta$ :

$$
\hat{\vartheta}=\operatorname{argmax}_{\vartheta \in \Theta} \sum_{i=1}^{n}\left\{\log \hat{f}_{\hat{\varepsilon}(\vartheta)}\left(\frac{\Lambda_{\vartheta}\left(Y_{i}\right)-\hat{m}_{\vartheta}\left(X_{i}\right)}{\hat{\sigma}_{\vartheta}\left(X_{i}\right)}\right)+\log \Lambda_{\vartheta}^{\prime}\left(Y_{i}\right)-\log \hat{\sigma}_{\vartheta}\left(X_{i}\right)\right\} .
$$

In order to obtain an asymptotic i.i.d. representation and the asymptotic normality of the estimator $\hat{\vartheta}$, we need to introduce a number of notations. For any function $h_{\vartheta}$ we denote by $\dot{h}_{\vartheta}=\nabla_{\vartheta} h_{\vartheta}$ the vector of partial derivatives of $h_{\vartheta}$ with respect to the components of $\vartheta$. Let

$$
G_{n}(\vartheta)=\frac{1}{n} \sum_{i=1}^{n} g_{\vartheta}\left(X_{i}, Y_{i}\right)
$$

be the derivative of the log-likelihood given in (2.2) (divided by $n$ ) with respect to $\vartheta$, where

$$
\begin{aligned}
g_{\vartheta}\left(X_{i}, Y_{i}\right)= & \frac{f_{\varepsilon(\vartheta)}^{\prime}\left(\varepsilon_{i}(\vartheta)\right)}{f_{\varepsilon(\vartheta)}\left(\varepsilon_{i}(\vartheta)\right)}\left[\frac{\dot{\Lambda}_{\vartheta}\left(Y_{i}\right)-\dot{m}_{\vartheta}\left(X_{i}\right)}{\sigma_{\vartheta}\left(X_{i}\right)}-\left\{\Lambda_{\vartheta}\left(Y_{i}\right)-m_{\vartheta}\left(X_{i}\right)\right\} \frac{\dot{\sigma}_{\vartheta}\left(X_{i}\right)}{\sigma_{\vartheta}^{2}\left(X_{i}\right)}\right] \\
& +\frac{\dot{f}_{\varepsilon(\vartheta)}\left(\varepsilon_{i}(\vartheta)\right)}{f_{\varepsilon(\vartheta)}\left(\varepsilon_{i}(\vartheta)\right)}+\frac{\dot{\Lambda}_{\vartheta}^{\prime}\left(Y_{i}\right)}{\Lambda_{\vartheta}^{\prime}\left(Y_{i}\right)}-\frac{\dot{\sigma}_{\vartheta}\left(X_{i}\right)}{\sigma_{\vartheta}\left(X_{i}\right)}
\end{aligned}
$$

Then $G_{n}(\vartheta)$ converges in probability to $G(\vartheta)=E\left[g_{\vartheta}(X, Y)\right]$. We assume that $\vartheta_{0}$ is the unique zero of $G$ (see assumption (a7) in appendix A). The next theorem states the asymptotic normality of the estimator $\hat{\vartheta}$. The result shows that the variance of the estimator is the same as in the case where the nonparametric functions $m_{\vartheta}(x), \sigma_{\vartheta}(x)$ and $f_{\varepsilon(\vartheta)}(y)$ and their derivatives with respect to $\vartheta$ and $y$ would be known, which is quite remarkable. The cancellation of all terms derived from estimators of nuisance functions has been observed in other contexts where profile likelihood methods have been used. See e.g. Severini and Wong (1992) among others, where we can observe that the profile likelihood method internalizes the estimation cost associated with the nonparametric functions. The regularity conditions under which this result is valid are given in appendix A. 
Theorem 2.1 Assume (a1)-(ar) in Appendix A. Then,

$$
\hat{\vartheta}-\vartheta_{0}=-\Gamma^{-1} \frac{1}{n} \sum_{i=1}^{n} g_{\vartheta_{0}}\left(X_{i}, Y_{i}\right)+o_{P}\left(n^{-1 / 2}\right),
$$

and

$$
n^{1 / 2}\left(\hat{\vartheta}-\vartheta_{0}\right) \stackrel{d}{\rightarrow} N(0, \Sigma)
$$

where $\Sigma=\Gamma^{-1} \operatorname{Var}\left[g_{\vartheta_{0}}(X, Y)\right] \Gamma^{-1}$ and $\Gamma=\left.\nabla_{\vartheta} G(\vartheta)^{\top}\right|_{\vartheta=\vartheta_{0}}$.

The proof of this result can be found in the supplementary document.

\subsection{Estimation of regression and variance functions}

Once the transformation parameter vector $\vartheta_{0}$ is estimated, we can go back to the estimation of the regression function $m(x)$ and the variance function $\sigma^{2}(x)$. Define

$$
\hat{m}(x)=\hat{m}_{\hat{\vartheta}}(x) \quad \text { and } \quad \hat{\sigma}^{2}(x)=\hat{\sigma}_{\hat{\vartheta}}^{2}(x) .
$$

Under regularity conditions the estimation of $\vartheta_{0}$ has no influence on the asymptotic distribution of the centered and scaled estimators $\left(n h^{d}\right)^{1 / 2}(\hat{m}(x)-E[\hat{m}(x)])$ and $\left(n h^{d}\right)^{1 / 2}\left(\hat{\sigma}^{2}(x)-\right.$ $\left.E\left[\hat{\sigma}^{2}(x)\right]\right)$, since $\hat{\vartheta}$ has a parametric rate of convergence. Therefore, the estimators behave asymptotically as if the true $\vartheta_{0}$ would be known. Note, however, that the pre-estimation of $\vartheta_{0}$ influences the asymptotic distribution of the test statistic in Section 3 because the integrals $\int\left(\hat{m}_{\vartheta_{0}}-m\right) / \sigma d F_{X}$ and $\int\left(\hat{m}_{\hat{\vartheta}}-\hat{m}_{\vartheta_{0}}\right) / \sigma d F_{X}$ have the same $n^{1 / 2}$-rate of convergence (see terms $B_{n}$ and $C_{n}$ in the proof of Theorem 3.1) and a similar statement holds for the variance estimator.

\subsection{Estimation of the error distribution}

The last unknown component of our heteroscedastic transformation model (2.1) is the distribution $F_{\varepsilon}$ of the error term. Define the residuals as

$$
\hat{\varepsilon}_{i}=\hat{\varepsilon}_{i}(\hat{\vartheta})=\frac{\Lambda_{\hat{\vartheta}}\left(Y_{i}\right)-\hat{m}\left(X_{i}\right)}{\hat{\sigma}\left(X_{i}\right)} .
$$

The error distribution $F_{\varepsilon}(y)$ can now be estimated by the empirical distribution function of the $\hat{\varepsilon}_{i}$ 's:

$$
\hat{F}_{\hat{\varepsilon}}(y)=\frac{1}{n} \sum_{i=1}^{n} I\left\{\hat{\varepsilon}_{i} \leq y\right\},
$$

where $I$ denotes the indicator function. We postpone the study of the asymptotic properties of this estimator to the next section. In fact, in Section 3 we will study an estimator of the joint distribution of $X$ and $\varepsilon$, which includes the estimator $\hat{F}_{\hat{\varepsilon}}(y)$ as a special case. 


\section{Testing the validity of the model}

In this section we develop tests for validity of a heteroscedastic semiparametric transformation model. Let again $L=\left\{\Lambda_{\vartheta} \mid \vartheta \in \Theta\right\}$ be some parametric class of transformations, $\Theta$ some nonempty subset of $\mathbb{R}^{k}$. Our aim is to test the null hypothesis

$$
H_{0}: \exists \vartheta \in \Theta \text { such that } \frac{\Lambda_{\vartheta}(Y)-E\left[\Lambda_{\vartheta}(Y) \mid X\right]}{\left(\operatorname{Var}\left(\Lambda_{\vartheta}(Y) \mid X\right)\right)^{1 / 2}} \perp X .
$$

If the null hypothesis is valid then there exists some transformation $\Lambda_{\vartheta_{0}} \in L$ with which one obtains a nonparametric location-scale model as in (2.1). Note that we want to test the appropriateness of the parametric family of transformations. So, our test is a goodness-of-fit test for the chosen parametric family. We do not test whether data is from a transformation model or not. If we reject $H_{0}$ it could be that the data is from a transformation model but that the true transformation does not belong to the family $L$ under our consideration.

\subsection{The test statistics and asymptotic distributions under $H_{0}$}

Let $\hat{\vartheta}$ be some estimator for the true parameter $\vartheta_{0}$ under $H_{0}$ such that a linear expansion

$$
\hat{\vartheta}-\vartheta_{0}=\frac{1}{n} \sum_{i=1}^{n} g_{\vartheta_{0}}\left(X_{i}, Y_{i}\right)+o_{P}\left(\frac{1}{\sqrt{n}}\right)
$$

is valid under $H_{0}$, where $E\left[g_{\vartheta_{0}}\left(X_{i}, Y_{i}\right)\right]=0, E\left[\left\|g_{\vartheta_{0}}\left(X_{i}, Y_{i}\right)\right\|^{2}\right]<\infty$. We have shown in Theorem 2.1 that such an expansion is valid for the profile likelihood estimator under some regularity conditions. Now denote by $\hat{F}_{X, \hat{\varepsilon}}$ the joint empirical distribution function of covariates and residuals, i. e.

$$
\hat{F}_{X, \hat{\varepsilon}}(x, y)=\frac{1}{n} \sum_{i=1}^{n} I\left\{X_{i} \leq x, \hat{\varepsilon}_{i} \leq y\right\},
$$

where $\leq$ for vectors is meant componentwise. We consider test statistics based on the estimated independence empirical process

$$
S_{n}=\sqrt{n}\left(\hat{F}_{X, \hat{\varepsilon}}-\hat{F}_{X} \hat{F}_{\hat{\varepsilon}}\right)
$$

where $\hat{F}_{X}(x)=n^{-1} \sum_{i=1}^{n} I\left\{X_{i} \leq x\right\}$ and $\hat{F}_{\hat{\varepsilon}}(y)=n^{-1} \sum_{i=1}^{n} I\left\{\hat{\varepsilon}_{i} \leq y\right\}$. 
Theorem 3.1 Assume (a1), (a2) and (A1)-(A8) from appendix A. Then, under $H_{0}$, we have the asymptotic expansion:

$$
\begin{aligned}
\hat{F}_{X, \hat{\varepsilon}}(x, y)= & \frac{1}{n} \sum_{i=1}^{n}\left(I\left\{X_{i} \leq x\right\}\left(I\left\{\varepsilon_{i} \leq y\right\}+f_{\varepsilon}(y)\left(\varepsilon_{i}+\frac{y}{2}\left(\varepsilon_{i}^{2}-1\right)\right)\right)\right. \\
& \left.+E\left[\left.\nabla_{\vartheta} F_{\varepsilon(\vartheta) \mid X}(y \mid X)\right|_{\vartheta=\vartheta_{0}} I\{X \leq x\}\right]^{\top} g_{\vartheta_{0}}\left(X_{i}, Y_{i}\right)\right)+o_{P}\left(n^{-1 / 2}\right)
\end{aligned}
$$

uniformly with respect to $x \in R_{X}, y \in \mathbb{R}$.

The proof is given in appendix B. From the theorem one directly obtains the following result for the residual based empirical distribution function defined in Section 2.3.

Corollary 3.2 Under the assumptions of Theorem 3.1, we have the asymptotic expansion:

$$
\begin{aligned}
\hat{F}_{\hat{\varepsilon}}(y)= & \frac{1}{n} \sum_{i=1}^{n}\left(I\left\{\varepsilon_{i} \leq y\right\}+f_{\varepsilon}(y)\left(\varepsilon_{i}+\frac{y}{2}\left(\varepsilon_{i}^{2}-1\right)\right)\right) \\
& \left.+E\left[\left.\nabla_{\vartheta} F_{\varepsilon(\vartheta) \mid X}(y \mid X)\right|_{\vartheta=\vartheta_{0}}\right]^{\top} g_{\vartheta_{0}}\left(X_{i}, Y_{i}\right)\right)+o_{P}\left(n^{-1 / 2}\right)
\end{aligned}
$$

uniformly with respect to $y \in \mathbb{R}$. The process $\sqrt{n}\left(\hat{F}_{\hat{\varepsilon}}-F_{\varepsilon}\right)$ converges weakly in $\ell^{\infty}(\mathbb{R})$ to a centered Gaussian process.

This corollary generalizes the main results by Heuchenne et al. (2015) who consider estimation of the error distribution in a homoscedastic transformation model. The asymptotic expansion directly follows from Theorem 3.1. The proof of weak convergence is analogous to the proof of Corollary 3.3 below and thus omitted.

Using that the dominating term in this expansion has expectation $F_{\varepsilon}(y)$ and applying that $\hat{F}_{X}=F_{X}+O_{p}\left(n^{-1 / 2}\right)$ one straightforwardly obtains the following expansion for the process $S_{n}$ defined in (3.3):

$$
S_{n}(x, y)=\frac{1}{\sqrt{n}} \sum_{i=1}^{n} \psi_{x, y, \vartheta_{0}}\left(X_{i}, Y_{i}\right)+o_{P}(1)
$$

uniformly with respect to $x \in R_{X}, y \in \mathbb{R}$, where

$$
\begin{aligned}
\psi_{x, y, \vartheta_{0}}\left(X_{i}, Y_{i}\right)= & \left(I\left\{X_{i} \leq x\right\}-F_{X}(x)\right)\left(I\left\{\varepsilon_{i} \leq y\right\}-F_{\varepsilon}(y)+f_{\varepsilon}(y)\left(\varepsilon_{i}+\frac{y}{2}\left(\varepsilon_{i}^{2}-1\right)\right)\right) \\
& +E\left[\left.\nabla_{\vartheta} F_{\varepsilon(\vartheta) \mid X}(y \mid X)\right|_{\vartheta=\vartheta_{0}}\left(I\{X \leq x\}-F_{X}(x)\right)\right]^{\top} g_{\vartheta_{0}}\left(X_{i}, Y_{i}\right) .
\end{aligned}
$$


Corollary 3.3 Under the assumptions of Theorem 3.1, the process $S_{n}$ converges weakly in $\ell^{\infty}\left(R_{X} \times \mathbb{R}\right)$ to a centered Gaussian process $S$ with covariance $\operatorname{Cov}(S(x, y), S(u, z))=$ $E\left[\psi_{x, y, \vartheta_{0}}(X, Y) \psi_{u, z, \vartheta_{0}}(X, Y)\right]$.

The proof is given in appendix B. Let $\Psi$ denote some continuous functional from $\ell^{\infty}\left(R_{X} \times \mathbb{R}\right)$ to $\mathbb{R}$, e.g. $\Psi(s)=\sup _{x, y}|s(x, y)|$ for a Kolmogorov-Smirnov test. Then we reject $H_{0}$ with nominal level $\alpha$ if $T_{n}=\Psi\left(S_{n}\right)$ exceeds a critical value $c_{\alpha}$. A bootstrap approximation of $c_{\alpha}$ is given in Section 3.2. In our simulations, we use the KolmogorovSmirnov and Cramér-von Mises test statistics:

$$
\begin{aligned}
T_{n, K S} & =\sqrt{n} \sup _{x, y}\left|\hat{F}_{X, \hat{\varepsilon}}(x, y)-\hat{F}_{X}(x) \hat{F}_{\hat{\varepsilon}}(y)\right| \\
T_{n, C M} & =n \iint\left(\hat{F}_{X, \hat{\varepsilon}}(x, y)-\hat{F}_{X}(x) \hat{F}_{\hat{\varepsilon}}(y)\right)^{2} d \hat{F}_{X}(x) d \hat{F}_{\hat{\varepsilon}}(y) .
\end{aligned}
$$

\subsection{Bootstrap approximation of the critical value}

Since the asymptotic distributions of the test statistics depend in a complicated way on unknown quantities, we suggest to apply a bootstrap procedure to approximate the critical values. To this end let $\eta_{1}^{*}, \ldots, \eta_{n}^{*}$ be drawn with replacement from standardized residuals $\tilde{\varepsilon}_{1}, \ldots, \tilde{\varepsilon}_{n}$, where

$$
\tilde{\varepsilon}_{i}=\frac{\hat{\varepsilon}_{i}-n^{-1} \sum_{k=1}^{n} \hat{\varepsilon}_{k}}{\left(n^{-1} \sum_{j=1}^{n}\left(\hat{\varepsilon}_{j}-n^{-1} \sum_{k=1}^{n} \hat{\varepsilon}_{k}\right)\right)^{1 / 2}}, \quad i=1, \ldots, n .
$$

Let further $\xi_{1}, \ldots, \xi_{n}$ denote independent standard normally distributed random variables, independent of the original sample $\mathcal{Y}_{n}=\left\{\left(X_{1}, Y_{1}\right), \ldots,\left(X_{n}, Y_{n}\right)\right\}$, and let $a_{n}$ be some positive smoothing parameter. Define bootstrap errors as $\varepsilon_{i}^{*}=\eta_{i}^{*}+a_{n} \xi_{i}$. Note that methods based on residual empirical processes require smoothing of the bootstrap errors, cf. Neumeyer (2009b), among others. It is easily seen that, conditionally on $\mathcal{Y}_{n}, \varepsilon_{i}^{*}$ has a smooth distribution function

$$
\tilde{F}_{\hat{\varepsilon}}(y)=\frac{1}{n} \sum_{j=1}^{n} \Phi\left(\frac{y-\tilde{\varepsilon_{j}}}{a_{n}}\right)
$$

where $\Phi$ denotes the standard normal distribution function.

Now generate $X_{i}^{*}$ from $\hat{F}_{X}$ and define

$$
Y_{i}^{*}=\Lambda_{\hat{\vartheta}}^{-1}\left(Z_{i}^{*}\right), \text { where } Z_{i}^{*}=\hat{m}\left(X_{i}^{*}\right)+\hat{\sigma}\left(X_{i}^{*}\right) \varepsilon_{i}^{*}, \quad i=1, \ldots, n
$$


The bootstrap sample is $\left(X_{i}^{*}, Y_{i}^{*}\right), i=1, \ldots, n$, and fulfills $H_{0}$ by construction. To see this let $E_{n}^{*}$ and $\operatorname{Var}_{n}^{*}$ denote the expectation and variance with respect to the conditional distribution $P\left(\cdot \mid \mathcal{Y}_{n}\right)$. Then $E_{n}^{*}\left[\varepsilon_{i}^{*} \mid X_{i}^{*}\right] \equiv 0$ and $\operatorname{Var}_{n}^{*}\left(\varepsilon_{i}^{*} \mid X_{i}^{*}\right) \equiv 1+a_{n}^{2}$ and thus

$$
\frac{\Lambda_{\hat{\vartheta}}\left(Y_{i}^{*}\right)-E_{n}^{*}\left[\Lambda_{\hat{\vartheta}}\left(Y_{i}^{*}\right) \mid X_{i}^{*}\right]}{\left(\operatorname{Var}_{n}^{*}\left(\Lambda_{\hat{\vartheta}}\left(Y_{i}^{*}\right) \mid X_{i}^{*}\right)\right)^{1 / 2}}=\frac{\varepsilon_{i}^{*}}{\left(1+a_{n}^{2}\right)^{1 / 2}} \perp X_{i}^{*}
$$

(given $\mathcal{Y}_{n}$ ). Let $T_{n}$ denote the test statistic based on the original sample and let $T_{n}^{*}$ be the one based on the bootstrap sample. Then $H_{0}$ is rejected whenever $T_{n}>c_{n, \alpha}$, where $P\left(T_{n}^{*}>c_{n, \alpha} \mid \mathcal{Y}_{n}\right)=1-\alpha$. The critical value $c_{n, \alpha}$ is estimated by the $\lfloor B(1-\alpha)\rfloor$-largest bootstrap test statistic obtained from $B$ replications of the bootstrap data generation.

\subsection{Remarks on consistency of the proposed tests}

We consider the hypothesis test developed in Section 3.1 when using the profile likelihood estimator $\hat{\vartheta}$ suggested in Section 2.1. With the notations used before let

$$
p_{\vartheta}(y \mid x)=f_{\varepsilon(\vartheta)}\left(\frac{\Lambda_{\vartheta}(y)-m_{\vartheta}(x)}{\sigma_{\vartheta}(x)}\right) \frac{\Lambda_{\vartheta}^{\prime}(y)}{\sigma_{\vartheta}(x)} .
$$

Note that $p_{\vartheta}$ is a conditional density, and a consistent estimator (under mild regularity conditions) of the log-likelihood

$$
L_{\vartheta}=\log \left(\prod_{i=1}^{n} p_{\vartheta}\left(Y_{i} \mid X_{i}\right)\right)
$$

is maximized in order to obtain the profile likelihood estimator of the transformation parameter $\vartheta \in \Theta$ (see (2.2)). Now, consider the alternative $H_{1}$, which states that there exists no parameter $\vartheta \in \Theta$ such that $p_{\vartheta}(\cdot \mid x)$ is the conditional density of $Y$, given $X=x$. Then $L_{\vartheta} / n$ estimates the expectation

$$
E\left[\log p_{\vartheta}\left(Y_{i} \mid X_{i}\right)\right]=\iint\left(\log p_{\vartheta}(y \mid x)\right) f_{Y \mid X}(y \mid x) d y d F_{X}(x)
$$

and thus $\hat{\vartheta}$ estimates the value $\vartheta_{1} \in \Theta$ which minimizes the expected Kullback-Leibler divergence of the conditional densities $f_{Y \mid X}$ and $p_{\vartheta}$, i. e.

$$
\iint\left(\log \frac{f_{Y \mid X}(y \mid x)}{p_{\vartheta}(y \mid x)}\right) f_{Y \mid X}(y \mid x) d y d F_{X}(x) .
$$

Thus $\hat{F}_{X, \hat{\varepsilon}}$ as defined in section 3.1 estimates the joint distribution of $X$ and $\varepsilon\left(\vartheta_{1}\right)=$ $\left(\Lambda_{\vartheta_{1}}(Y)-E\left[\Lambda_{\vartheta_{1}}(Y) \mid X\right]\right) /\left(\operatorname{Var}\left(\Lambda_{\vartheta_{1}}(Y) \mid X\right)\right)^{1 / 2}$. Since under $H_{1}$ the distribution of $\varepsilon\left(\vartheta_{1}\right)$ depends on $X$, it follows that, e.g., a Kolmogorov-Smirnov test statistic $T_{n}=\sup _{x, y}\left|S_{n}(x, y)\right|$ converges to infinity. Thus any test that rejects $H_{0}$ whenever $T_{n}$ exceeds some constant $c_{\alpha}$ is consistent. 


\subsection{The homoscedastic transformation model}

Let independent copies of $(X, Y)$ be observed and a parametric class of transformations $\left\{\Lambda_{\vartheta} \mid \vartheta \in \Theta\right\}$ be given. Then tests for the null hypothesis

$$
H_{0}: \exists \vartheta \in \Theta \text { such that } \Lambda_{\vartheta}(Y)-E\left[\Lambda_{\vartheta}(Y) \mid X\right] \perp X
$$

are also of interest. The validity of the null hypothesis means that a nonparametric location model

$$
\Lambda_{\vartheta_{0}}(Y)=m(X)+\varepsilon, \quad \varepsilon \perp X
$$

with $m(x)=E\left[\Lambda_{\vartheta_{0}}(Y) \mid X=x\right]$ describes the data for some $\vartheta_{0} \in \Theta$. Tests for model validity can be derived similarly as in the heteroscedastic case in an obvious manner. An estimator for the transformation parameter analogous to Linton et al. (2008) can be applied where the additive regression estimator is replaced by a purely nonparametric local polynomial estimator. The residuals are then defined as $\hat{\varepsilon}=\Lambda_{\hat{\vartheta}}(Y)-\hat{m}_{\hat{\vartheta}}(X)$. Under slightly weaker assumptions than those stated in Appendix A, similar asymptotic results to those in Section 3.1 can be derived. Additionally, we can use the simplification of the bootstrap in Section 3.2 to implement the test for the validity of (3.9) replacing $\tilde{\varepsilon}_{i}$ in (3.7) with $\tilde{\varepsilon}_{i}=\hat{\varepsilon}_{i}-n^{-1} \sum_{k=1}^{n} \hat{\varepsilon}_{k}$, and $Z_{i}^{*}$ in (3.8) with $Z_{i}^{*}=\hat{m}\left(X_{i}^{*}\right)+\varepsilon_{i}^{*}$.

\section{Numerical simulations}

In this section, we carry out three different simulation studies. All computations are done with R (R Development Core Team 2015). Firstly, we illustrate the finite sample performance of the estimator $\hat{\vartheta}$ of the transformation parameter in (2.4). Secondly, we study the performance of the proposed test for checking homoscedasticity under some transformation when it is implemented via the bootstrap described in Section 3.4. Finally, we verify how well the test in Section 3.1 is able to test the assumption of a heteroscedastic transformation structure, when the true model gradually deviates from a heteroscedastic transformation model.

Throughout all simulations, we consider the Yeo-Johnson family of transformations:

$$
\Lambda_{\vartheta}(y)=\left\{\begin{array}{ll}
\left\{(y+1)^{\vartheta}-1\right\} / \vartheta & y \geq 0, \vartheta \neq 0 \\
\log (y+1) & y \geq 0, \vartheta=0 \\
-\left\{(-y+1)^{2-\vartheta}-1\right\} /(2-\vartheta) & y<0, \vartheta \neq 2 \\
-\log (-y+1) & y<0, \vartheta=2
\end{array},\right.
$$


which was proposed by Yeo and Johnson (2000) as a generalization of the Box-Cox family of transformations. Concerning the estimation of the transformation parameter, we use the normal kernel whenever a kernel function is necessary. To estimate $m(\cdot)$ and $\sigma(\cdot)$, we use the local linear estimator $(p=1)$ using the $\mathrm{R}$ package $n p$ (See Hayfield and Racine 2008). The bandwidth is chosen by the direct plug-in methodology described by Ruppert, Sheather and Wand (1995). For estimation of $f_{\varepsilon(\vartheta)}(\cdot)$, we use the bandwidth obtained from the method of Sheather and Jones (1991). All these bandwidth selection methods are implemented in the R package KernSmooth (See Wand 2015).

\subsection{Estimation of heteroscedastic transformation parameter}

To see how the estimator $\hat{\vartheta}$ in (2.4) works in practice, we generate data from the following heteroscedastic transformation model:

$$
\Lambda_{\vartheta_{0}}\left(Y_{i}\right)=m\left(X_{i}\right)+\sigma\left(X_{i}\right) \varepsilon_{i}, i=1, \cdots, n,
$$

where $m(x)=\exp (x)+1.5$ and $\sigma(x)=1+a(x-1)$. Moreover, $X_{1}, \ldots, X_{n}$ are independent and uniformly distributed on $[0,1], \varepsilon_{1}, \ldots, \varepsilon_{n}$ are independent standard normal random variables, and $X_{i}$ and $\varepsilon_{i}$ are independent. We let $\theta_{0}$ be zero here and whenever this model is used further on. For various values of $a$ and $n$, we calculate $\hat{\vartheta}$ from 200 samples of size $n=100,200$ and 400, and compute

$$
\operatorname{MEAN}=\frac{1}{200} \sum_{j=1}^{200} \hat{\vartheta}^{(j)} \text { and MSE }=\frac{1}{200} \sum_{j=1}^{200}\left(\hat{\vartheta}^{(j)}-\vartheta_{0}\right)^{2}
$$

where $\hat{\vartheta}^{(j)}$ is the estimate of $\vartheta_{0}$ from the $j$ th sample. The results are given in Table 1 . For various values of $a$, we observe that both the bias and the mean squared error of the estimator decrease as the sample size increases, which suggests the consistency of the

\begin{tabular}{|c|c|c|c|c|c|c|}
\hline & \multicolumn{2}{|c|}{$n=100$} & \multicolumn{2}{|c|}{$n=200$} & \multicolumn{2}{|c|}{$n=400$} \\
\hline & MEAN & MSE & MEAN & MSE & MEAN & MSE \\
\hline$a=0.5$ & 0.085 & 0.198 & 0.035 & 0.117 & 0.026 & 0.062 \\
\hline$a=0.75$ & 0.077 & 0.200 & 0.048 & 0.090 & 0.008 & 0.053 \\
\hline$a=1$ & 0.056 & 0.228 & 0.074 & 0.121 & -0.009 & 0.066 \\
\hline
\end{tabular}
estimator.

Table 1: The bias and mean squared error of the estimator $\hat{\vartheta}$ for $n=100,200$ and 400 . 


\subsection{Testing for homoscedastic transformation models}

To verify the performance of the test proposed in Section 3.4 regarding the assumption of a homoscedastic transformation model, we reuse model (4.1). Note that the degree of heteroscedasticity decreases as the value of $a$ gets closer to 0 and model (4.1) becomes a homoscedastic transformation model when $a=0$, which satisfies the null hypothesis (3.9). We investigate how the test behaves as the value of $a$ increases from 0 to 1.

With regard to the test statistics, we consider the Kolmogorov-Smirnov and Cramérvon Mises test statistics in (3.5). To find the critical value for the proposed tests, we use 200 bootstrap replications for each sample. For the smooth bootstrap described in Section 3.4, we set $a_{n}$ to $0.5 n^{-1 / 4}$ as in Neumeyer (2009b). In that reference the validity of a smooth residual bootstrap procedure for the residual-based empirical process (in a model without transformation) was shown rigorously under some conditions on the relationship between the bandwidths $h_{1}$ and $a_{n}$ when $d=1$ (see assumption A.6 in the reference). They basically mean

$$
\frac{n h_{1} a_{n}^{2+\Delta_{1}}}{\log \left(h_{1}^{-1}\right)} \rightarrow \infty, \quad h_{1}=o\left(a_{n}^{1+\Delta_{2}}\right) \text { for some small positive } \Delta_{1}, \Delta_{2} .
$$

We conjecture that combining the methods of proof in the reference and in the paper at hand we could prove the validity of the smooth residual bootstrap for the transformation model under similar conditions. For $a_{n} \sim n^{-1 / 4}$ the conditions in (4.2) amount to $n h_{1}^{2} \omega_{1, n} \rightarrow \infty, n h_{1}^{4} \omega_{2, n} \rightarrow 0$ for some sequences $\omega_{1, n} \rightarrow 0$ and $\omega_{2, n} \rightarrow \infty$ slowly. In comparison our bandwidth conditions (a2) for $p=d=1$ say $n h_{1}^{3+\delta} \rightarrow \infty, n h_{1}^{4} \rightarrow 0$, so they do not contradict the conditions for the bootstrap.

Table 2 shows the results for the test implemented via the bootstrap described in Section 3.4. We see that the size of the test is somewhat too low, but the power grows to one as the parameter $a$ measuring the degree of heteroscedasticity gets larger. One notable feature of the results is that the power does not change much until the degree of heteroscedasticity reaches a certain level and then starts to increase rapidly. To explain this peculiar behavior, we show in Figure 1 four plots using data of size $n=200$ from model (4.1). These plots are given for two values of $a$, and compare the regression function based on the true parameter $\vartheta_{0}$ with the one based on the estimator $\hat{\vartheta}$.

When $a \neq 0$, the estimator $\hat{\vartheta}$ is not consistent due to the misspecification of the heteroscedastic error structure, and instead targets the pseudo-true parameter $\vartheta^{*} \neq \vartheta_{0}$ which maximizes

$$
P L(\vartheta)=E\left(\log f_{\varepsilon_{\vartheta}}\left(\Lambda_{\vartheta}(Y)-m_{\vartheta}(X)\right)+\log \Lambda_{\vartheta}^{\prime}(Y)\right)
$$


$\mathrm{a}=0.5$

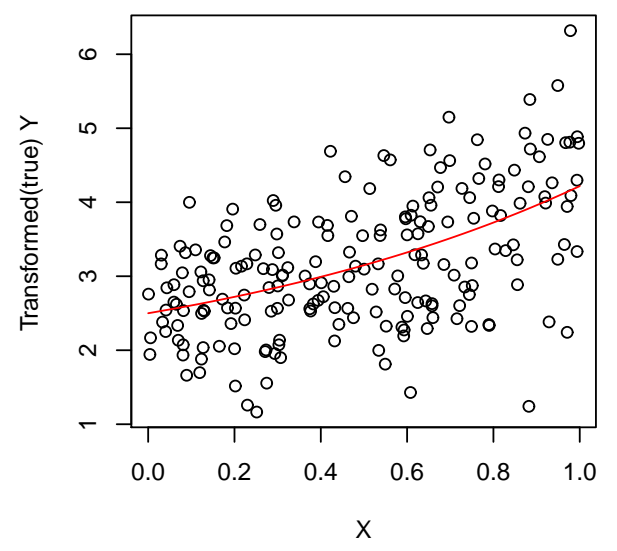

$a=1$

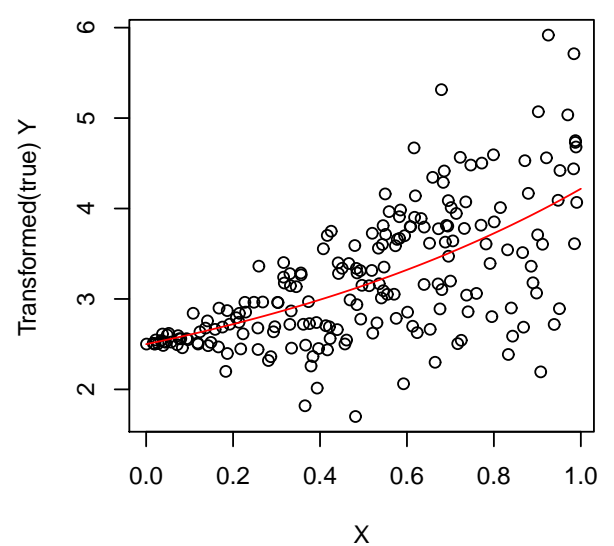

$a=0.5$

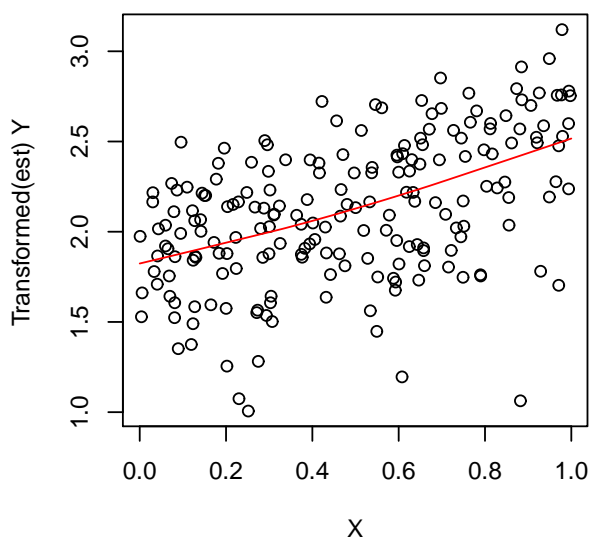

$a=1$

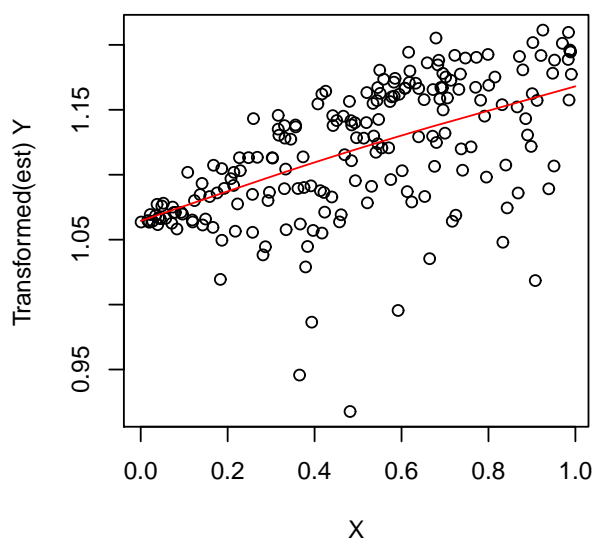

Figure 1: Plot of $\Lambda_{\vartheta=\vartheta_{0}}\left(Y_{i}\right)$ versus $X_{i}$ (left panel), and $\Lambda_{\vartheta=\hat{\vartheta}}\left(Y_{i}\right)$ versus $X_{i}$ (right panel), when $a=0.5$ (upper panel) and $a=1$ (lower panel). The curves $m_{\vartheta_{0}}(\cdot)$ (left) and $m_{\hat{\vartheta}}(\cdot)$ (right) are indicated in red.

where $m_{\vartheta}(x)=E\left(\Lambda_{\vartheta}(Y) \mid X=x\right)$ and $\varepsilon_{\vartheta}=\Lambda_{\vartheta}(Y)-m_{\vartheta}(X)$. This pseudo-true parameter has the interpretation that the corresponding homoscedastic model is the best approximation to the true heteroscedastic transformation model. So when the degree of heteroscedasticity is moderate, it is possible that the data look like data coming from a homoscedastic transformation model with transformation parameter $\hat{\vartheta}$ (see the upper right panel of Figure 1). In this case, our test is not able to detect the violation of assumption (3.9) well, and behaves similarly as if the null hypothesis is true. However, when the degree of heteroscedasticity becomes severe, the data cannot be considered anymore to come from a homoscedastic transformation model, and it becomes possible to detect the 


\begin{tabular}{|c|c|c|c|c|c|c|c|c|}
\hline & \multicolumn{4}{|c|}{$n=100$} & \multicolumn{4}{|c|}{$n=200$} \\
\hline & \multicolumn{2}{|c|}{$\alpha=0.05$} & \multicolumn{2}{|c|}{$\alpha=0.1$} & \multicolumn{2}{|c|}{$\alpha=0.05$} & \multicolumn{2}{|c|}{$\alpha=0.1$} \\
\hline & KS & $\mathrm{CM}$ & $\mathrm{KS}$ & $\mathrm{CM}$ & $\mathrm{KS}$ & $\mathrm{CM}$ & $\mathrm{KS}$ & $\mathrm{CM}$ \\
\hline$a=0$ & 0.040 & 0.035 & 0.075 & 0.065 & 0.045 & 0.045 & 0.070 & 0.085 \\
\hline$a=0.5$ & 0.085 & 0.125 & 0.125 & 0.160 & 0.110 & 0.165 & 0.180 & 0.240 \\
\hline$a=0.75$ & 0.340 & 0.460 & 0.445 & 0.510 & 0.545 & 0.690 & 0.670 & 0.775 \\
\hline$a=1$ & 0.910 & 0.980 & 0.965 & 0.980 & 0.995 & 1.000 & 1.000 & 1.000 \\
\hline
\end{tabular}

Table 2: The power of the test for verifying the validity of a homoscedastic transformation structure. The power is calculated based on 200 samples. The null hypothesis is satisfied for $a=0$.

violation through the dependence between $X$ and $\hat{\varepsilon}$ (see the right lower panel of Figure 1). This feature is different from what was observed in testing for homoscedasticity in regression settings without transformation, such as in Neumeyer (2009a).

Since our testing procedure involves estimation of many parameters and functions, one may be interested in how the selection of the smoothing parameters for these estimators affects the performance of the proposed tests. First, we investigate the impact of the choice of $a_{n}$ on the test. We reran the simulation for Table 2 but with other choices of $a_{n}$ : $a_{n}=0.25 n^{-1 / 4}, n^{-1 / 4}$, which produces Table 3 . Table 3 suggests that the performance (level or power) of the test is not so sensitive to the choice of $a_{n}$. Second, we investigate the impact of bandwidth selection on the behavior of the tests. Concerning the test for homoscedastic transformation models, we need three bandwidths. To calculate the profile likelihood, we use two bandwidths for $\hat{m}_{\vartheta}$ and $\hat{f}_{\varepsilon(\vartheta)}$. Once $\hat{\vartheta}$ is obtained, we need another bandwidth to calculate the residual $\hat{\varepsilon}_{i}=\Lambda_{\hat{\vartheta}}\left(Y_{i}\right)-\hat{m}\left(X_{i}\right)$. In our simulations, all these bandwidths are chosen as the optimal bandwidths in terms of MISE using the methods of Ruppert et al. (1995) and Sheather and Jones (1991). In order to see the impact of bandwidth selection, we use half of the optimal bandwidth or twice the optimal bandwidth whenever the bandwidth selection is necessary and check the level and power with such bandwidth. The results are summarized in Table 4. As can be seen in Table 4, we observe that the level and power is not so sensitive to the choice of the bandwidths, which makes our procedure applicable in practice. 


\begin{tabular}{|c|c|c|c|c|c|c|c|c|c|}
\hline \multirow[b]{3}{*}{$a_{n}$} & \multirow[b]{3}{*}{$a$} & \multicolumn{4}{|c|}{$n=100$} & \multicolumn{4}{|c|}{$n=200$} \\
\hline & & \multicolumn{2}{|c|}{$\alpha=0.05$} & \multicolumn{2}{|c|}{$\alpha=0.1$} & \multicolumn{2}{|c|}{$\alpha=0.05$} & \multicolumn{2}{|c|}{$\alpha=0.1$} \\
\hline & & $\mathrm{KS}$ & $\mathrm{CM}$ & $\mathrm{KS}$ & $\mathrm{CM}$ & $\mathrm{KS}$ & $\mathrm{CM}$ & $\mathrm{KS}$ & $\mathrm{CM}$ \\
\hline \multirow{4}{*}{$0.25 n^{-1 / 4}$} & $a=0$ & 0.025 & 0.030 & 0.055 & 0.055 & 0.040 & 0.040 & 0.065 & 0.085 \\
\hline & $a=0.5$ & 0.080 & 0.120 & 0.130 & 0.155 & 0.120 & 0.165 & 0.180 & 0.245 \\
\hline & $a=0.75$ & 0.300 & 0.440 & 0.415 & 0.510 & 0.555 & 0.690 & 0.645 & 0.785 \\
\hline & $a=1$ & 0.910 & 0.975 & 0.955 & 0.975 & 0.995 & 1.000 & 1.000 & 1.000 \\
\hline \multirow{4}{*}{$n^{-1 / 4}$} & $a=0$ & 0.035 & 0.025 & 0.090 & 0.070 & 0.040 & 0.070 & 0.080 & 0.090 \\
\hline & $a=0.5$ & 0.095 & 0.115 & 0.170 & 0.170 & 0.130 & 0.150 & 0.170 & 0.240 \\
\hline & $a=0.75$ & 0.335 & 0.450 & 0.425 & 0.520 & 0.550 & 0.660 & 0.640 & 0.740 \\
\hline & $a=1$ & 0.905 & 0.975 & 0.955 & 0.980 & 0.990 & 1.000 & 1.000 & 1.000 \\
\hline
\end{tabular}

Table 3: The power of the test for verifying the validity of a homoscedastic transformation structure when different bandwidths $a_{n}$ are used for the smooth bootstrap. The power is calculated based on 200 samples. The null hypothesis is satisfied for $a=0$.

\begin{tabular}{|c|c|c|c|c|c|c|c|c|c|}
\hline & \multirow[b]{3}{*}{ bandwidth } & \multicolumn{4}{|c|}{$n=100$} & \multicolumn{4}{|c|}{$n=200$} \\
\hline & & \multicolumn{2}{|c|}{$\alpha=0.05$} & \multicolumn{2}{|c|}{$\alpha=0.1$} & \multicolumn{2}{|c|}{$\alpha=0.05$} & \multicolumn{2}{|c|}{$\alpha=0.1$} \\
\hline & & KS & $\mathrm{CM}$ & $\mathrm{KS}$ & $\mathrm{CM}$ & $\mathrm{KS}$ & $\mathrm{CM}$ & $\mathrm{KS}$ & $\mathrm{CM}$ \\
\hline \multirow{3}{*}{$a=0$} & half & 0.030 & 0.040 & 0.085 & 0.065 & 0.080 & 0.070 & 0.010 & 0.011 \\
\hline & our choice & 0.040 & 0.035 & 0.075 & 0.065 & 0.045 & 0.045 & 0.070 & 0.085 \\
\hline & twice & 0.035 & 0.025 & 0.060 & 0.045 & 0.025 & 0.040 & 0.060 & 0.095 \\
\hline \multirow{3}{*}{$a=0.75$} & half & 0.285 & 0.360 & 0.395 & 0.495 & 0.590 & 0.700 & 0.690 & 0.780 \\
\hline & our choice & 0.340 & 0.460 & 0.455 & 0.510 & 0.545 & 0.690 & 0.670 & 0.775 \\
\hline & twice & 0.325 & 0.450 & 0.440 & 0.545 & 0.540 & 0.710 & 0.650 & 0.795 \\
\hline
\end{tabular}

Table 4: The power of the test for verifying the validity of a homoscedastic transformation structure when different bandwidths are used. The power is calculated based on 200 samples. The null hypothesis is satisfied for $a=0$. 


\subsection{Testing for heteroscedastic transformation models}

Finally, we illustrate how the test in Section 3.1 works to verify the assumption of a heteroscedastic transformation structure. For this purpose, we define two new transformation models. Basically, they are the same model as the model (4.1), except that the error distribution is defined by

Model A

$$
(\varepsilon \mid X=x) \sim \begin{cases}N\left(0,1^{2}\right) & \text { if } 0.5<x \leq 1 \\ (W-E(W)) / \sqrt{\operatorname{Var}(W)}, \text { where } W \sim S T(0,1, \alpha, \nu) & \text { if } 0 \leq x \leq 0.5\end{cases}
$$

$\underline{\text { Model B }}$

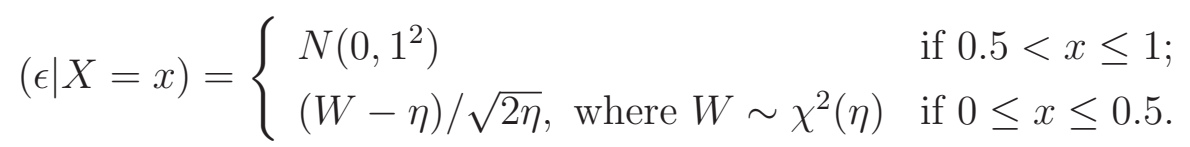

Here, $S T(\xi, \Omega, \alpha, \nu)$ is a skew- $t$ distribution with parameters $\xi, \Omega, \alpha$ and $\nu$ defined in Azzalini (2005). The parameter $\alpha$ controls the skewness of the distribution and the paramer $\nu$ controls kurtosis. Additionally, we set $\sigma(x)=x($ so $a=1)$. First, note that as $\nu \rightarrow \infty$ and $\alpha \rightarrow 0$, Model A converges to model (4.1) with $\sigma(x)=x$, which satisfies the assumption of a heteroscedastic transformation structure (the same thing happens as $\eta \rightarrow \infty$ in case of Model B). An additional remark regarding these models is that the first and second moments of the conditional error distribution given $X$ coincide with the respective moments under model (4.1). The parameters $\alpha, \nu$ and $\eta$ determine how much the model violates assumption (3.1). In our simulations, to see how the test performs when the true model gradually deviates from the assumption under the null hypothesis, we investigate the power function as $\nu$ changes from $\infty$ to 2.1 and then as $\alpha$ changes from 0 to 100 for Model A, and as $\eta$ changes from $\infty$ to 2 for Model B. Here, $\nu$ should be greater than 2 and $\eta$ should be equal to or greater than 2 otherwise the distribution of $W$ cannot be standardized due to variance explosion. For the smooth bootstrap described in Section 3.2 , we set $a_{n}$ to $0.5 n^{-1 / 4}$ and use 200 bootstrap replications.

Similarly to what was observed in the case of homoscedastic transformation models, we observe from Tables 5 and 6 that there is a threshold of difference in two component distributions in the error above which we can detect the violation of the assumption, and the power starts to grow beyond the threshold. Further, we observe that compared to Model A, the power of Model B is somewhat lower. The reason can be attributed to the flexibility of the heteroscedastic transformation model. Since it is a very flexible model, 


\begin{tabular}{|c|c|c|c|c|c|c|c|c|c|}
\hline & & \multicolumn{4}{|c|}{$n=100$} & \multicolumn{4}{|c|}{$n=200$} \\
\hline & & \multicolumn{2}{|c|}{$\alpha=0.05$} & \multicolumn{2}{|c|}{$\alpha=0.1$} & \multicolumn{2}{|c|}{$\alpha=0.05$} & \multicolumn{2}{|c|}{$\alpha=0.1$} \\
\hline & & KS & $\mathrm{CM}$ & $\mathrm{KS}$ & $\mathrm{CM}$ & $\mathrm{KS}$ & $\mathrm{CM}$ & KS & $\mathrm{CM}$ \\
\hline$\alpha=100$ & $\nu=2.1$ & 0.370 & 0.445 & 0.505 & 0.590 & 0.710 & 0.770 & 0.795 & 0.850 \\
\hline$\alpha=0$ & $\nu=2.1$ & 0.105 & 0.140 & 0.170 & 0.200 & 0.205 & 0.270 & 0.325 & 0.360 \\
\hline$\alpha=0$ & $\nu=5$ & 0.075 & 0.060 & 0.105 & 0.085 & 0.060 & 0.060 & 0.130 & 0.095 \\
\hline$\alpha=0$ & $\nu=\infty$ & 0.055 & 0.060 & 0.070 & 0.105 & 0.080 & 0.070 & 0.120 & 0.135 \\
\hline
\end{tabular}

Table 5: The power of the test for verifying the validity of a heterocedastic transformation structure from Model A. The power is calculated based on 200 samples. The null hypothesis is satisfied for $\alpha=0$ and $\nu=\infty$.

\begin{tabular}{|c|c|c|c|c|c|c|c|c|}
\hline & \multicolumn{4}{|c|}{$n=100$} & \multicolumn{4}{|c|}{$n=200$} \\
\hline & \multicolumn{2}{|c|}{$\alpha=0.05$} & \multicolumn{2}{|c|}{$\alpha=0.1$} & \multicolumn{2}{|c|}{$\alpha=0.05$} & \multicolumn{2}{|c|}{$\alpha=0.1$} \\
\hline & $\mathrm{KS}$ & $\mathrm{CM}$ & $\mathrm{KS}$ & $\mathrm{CM}$ & KS & $\mathrm{CM}$ & $\mathrm{KS}$ & $\mathrm{CM}$ \\
\hline$\eta=2$ & 0.215 & 0.220 & 0.285 & 0.310 & 0.325 & 0.355 & 0.455 & 0.440 \\
\hline$\eta=3$ & 0.100 & 0.165 & 0.175 & 0.270 & 0.155 & 0.220 & 0.270 & 0.295 \\
\hline$\eta=5$ & 0.090 & 0.095 & 0.140 & 0.150 & 0.120 & 0.125 & 0.190 & 0.200 \\
\hline$\eta=10$ & 0.050 & 0.065 & 0.091 & 0.125 & 0.100 & 0.105 & 0.140 & 0.190 \\
\hline$\eta=\infty$ & 0.065 & 0.060 & 0.105 & 0.115 & 0.045 & 0.055 & 0.100 & 0.100 \\
\hline
\end{tabular}

Table 6: The power of the test for verifying the validity of a heterocedastic transformation structure from Model B. The power is calculated based on 200 samples. The null hypothesis is satisfied for $\eta=\infty$.

unless the two component distributions in the error are strikingly different from each other, the generated data look like data coming from a heteroscedastic transformation model with appropriately chosen transformation parameter.

\subsection{Real data analysis}

To illustrate our method, we analyze the ultrasonic calibration data that can be found in the NIST/SEMATECH e-Handbook of Statistical Methods. The data is available on the website (http://www.itl.nist.gov/div898/handbook/pmd/section6/pmd631.htm). The response $Y$ is ultrasonic response and the predictor $X$ is metal distance. Concerning 
these data, it has been found in the e-book that the data seem to satisfy the assumption of homoscedastic transformation models with the square-root transformation of $Y$, which is $\sqrt{Y_{i}}=m\left(X_{i}\right)+\varepsilon_{i}, i=1, \ldots, n$. Hence, we would like to test whether our method can rediscover such validity without the information about the appropriate transformation. We consider the Box-Cox transformation family since the square-root transformation is included in the family and all the responses are positive. We calculate the $P$-values from the proposed test with various choices of $a_{n}$. For more accurate result, the number of bootstrap iterations is set to be 400 . The estimated transformation paper $\hat{\vartheta}$ was 0.436 , which is similar to 0.5. Further, the $P$-values in Table 7 suggest that the given data satisfy the assumption of the homoscedastic transformation models, which is consistent with the analysis of the previous study. Additionally, we compare the residual plots of the two regression models, $Y=m(X)+\varepsilon$ and $\Lambda_{\vartheta=0.436}(Y)=m^{\prime}(X)+\varepsilon^{\prime}$, where $\left\{\Lambda_{\vartheta}(\cdot)\right\}$ is the family of Box-Cox transformations. The plots (Figure 2) say that the the transformation of the response seems to stablize the variance function in the regression model.

\begin{tabular}{ccc}
\hline & \multicolumn{2}{c}{$P$-value } \\
\cline { 2 - 3 }$a_{n}$ & $\mathrm{KS}$ & $\mathrm{CM}$ \\
\hline $0.25 n^{-1 / 4}$ & 0.883 & 0.617 \\
$0.5 n^{-1 / 4}$ & 0.853 & 0.560 \\
$n^{-1 / 4}$ & 0.825 & 0.490 \\
\hline
\end{tabular}

Table 7: The calculated $P$-values for the validity of homoscedastic transformation models concerning the ultrasonic calibration data

\section{A Regularity conditions}

For the asymptotic normality of the estimator $\hat{\vartheta}$, we need the following regularity conditions:

(a1) $k$ is a symmetric probability density function supported on $[-1,1], k$ is $d+1$ times continuously differentiable, and $k^{(j)}( \pm 1)=0$ for $j=0, \ldots, d-1$.

(a2) $h_{j}(j=1, \ldots, d)$ satisfies $h_{j} / h_{0} \rightarrow c_{j}$ for some $0<c_{j}<\infty$ and some baseline bandwidth $h_{0}$ satisfying $n h_{0}^{2 p+2} \rightarrow 0$ for some $p \geq 3$, and $n h_{0}^{3 d+\delta} \rightarrow \infty$ for some small $\delta>0$. 

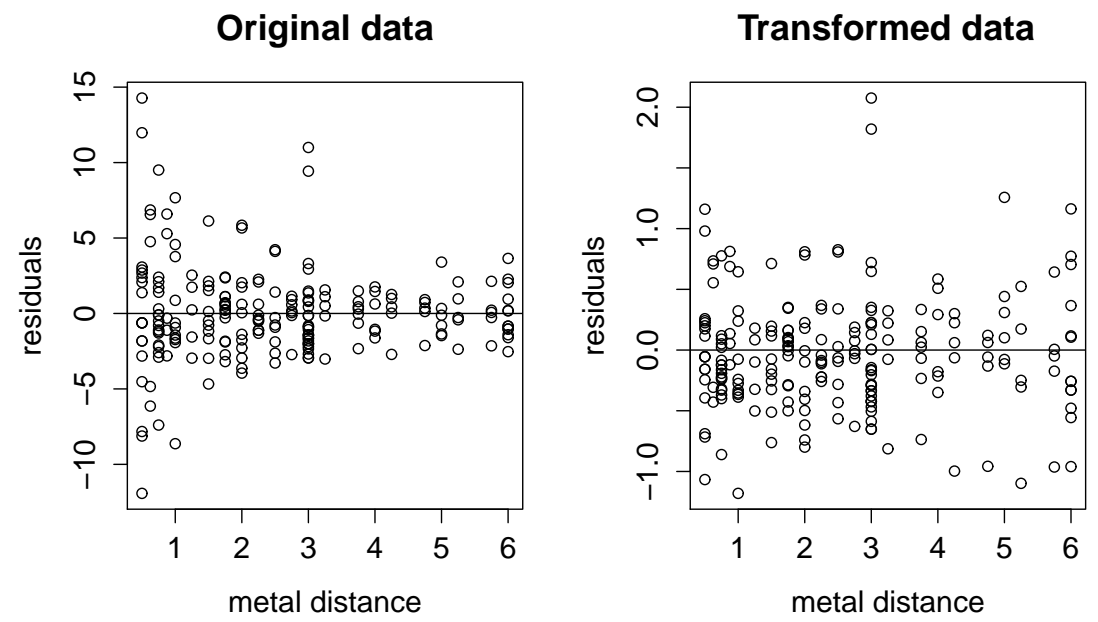

Figure 2: The residual plots of the two regression models, $Y=m(X)+\varepsilon$ and $\Lambda_{\vartheta=0.436}(Y)=$ $m^{\prime}(X)+\varepsilon^{\prime}$, where $\left\{\Lambda_{\vartheta}(\cdot)\right\}$ is the family of Box-Cox transformations.

(a3) The kernel $\ell$ is a symmetric, twice continuously differentiable function supported on $[-1,1], \int u^{s} \ell(u) d u=0$ for $s=1, \ldots, q-1$ and $\int u^{q} \ell(u) d u \neq 0$ for some $q \geq 4$. The bandwidth $g$ satisfies $n g^{6}(\log n)^{-2} \rightarrow \infty$ and $n g^{2 q} \rightarrow 0$.

(a4) The support $R_{X}$ of the covariate $X$ is a compact subset of $\mathbb{R}^{d}$, the distribution function $F_{X}$ is $2 d+1$-times continuously differentiable, $\inf _{x \in R_{X}} f_{X}(x)>0$ and $\inf _{x \in R_{X}} \sigma(x)>0$. Moreover, the functions $m_{\vartheta}(x), \dot{m}_{\vartheta}(x), \sigma_{\vartheta}(x)$ and $\dot{\sigma}_{\vartheta}(x)$ are $p+2$ times continuously differentiable with respect to the components of $x$ on $R_{X} \times \mathcal{N}\left(\vartheta_{0}\right)$, and all derivatives up to order $p+2$ are bounded uniformly in $(x, \vartheta) \in R_{X} \times \mathcal{N}\left(\vartheta_{0}\right)$, where $\mathcal{N}\left(\vartheta_{0}\right)$ is a neighborhood of $\vartheta_{0}$.

(a5) The transformation $\Lambda_{\vartheta}$ satisfies $\sup _{\vartheta \in \Theta, x \in R_{X}}\left\|E\left[\dot{\Lambda}_{\vartheta}(Y) \mid X=x\right]\right\|<\infty$, $\sup _{x \in R_{X}}\left\|E\left[\dot{\Lambda}_{\vartheta_{0}}^{4}(Y) \mid X=x\right]\right\|<\infty$, and the density function of $\left(\dot{\Lambda}_{\vartheta}(Y), X\right)$ exists and is continuous for all $\vartheta \in \Theta$. In addition, $\Lambda_{\vartheta}(y)$ is three times continuously differentiable with respect to $y$ and $\vartheta$, and there exists a $\delta>0$ such that

$$
E\left[\sup _{\vartheta^{\prime}:\left\|\vartheta^{\prime}-\vartheta\right\| \leq \delta}\left|\frac{\partial^{j+r}}{\partial y^{j} \partial \vartheta_{1}^{r_{1}} \ldots \partial \vartheta_{k}^{r_{k}}} \Lambda_{\vartheta^{\prime}}(Y)\right|\right]<\infty
$$

for all $\vartheta \in \Theta$ and all $0 \leq j+r \leq 3$, where $r=\sum_{i=1}^{k} r_{i}$.

(a6) The error term $\varepsilon$ has finite sixth moment and is independent of $X$. Moreover, the distribution $F_{\varepsilon(\vartheta)}(y)$ is three times continuously differentiable with respect to $y$ and 
$\vartheta$

$$
\sup _{y, \vartheta}\left|\frac{\partial^{j+r}}{\partial y^{j} \partial \vartheta_{1}^{r_{1}} \ldots \partial \vartheta_{k}^{r_{k}}} F_{\varepsilon(\vartheta)}(y)\right|<\infty
$$

for all $0 \leq j+\sum_{i=1}^{k} r_{i} \leq 2, \sup _{y}\left|y f_{\varepsilon}^{\prime}(y)\right|<\infty, \sup _{y}\left|y \dot{f}_{\varepsilon}^{\prime}(y)\right|<\infty$ and $\sup _{y}\left|y^{2} f_{\varepsilon}^{\prime \prime}(y)\right|<$ $\infty$. In addition, the conditional distribution $F_{\varepsilon(\vartheta) \mid X}(y \mid x)$ is three times continuously differentiable with respect to $y$ and $\vartheta$,

$$
\sup _{y, x, \vartheta}\left|\frac{\partial^{j+r}}{\partial y^{j} \partial \vartheta_{1}^{r_{1}} \ldots \partial \vartheta_{k}^{r_{k}}} F_{\varepsilon(\vartheta) \mid X}(y \mid x)\right|<\infty
$$

for all $0 \leq j+\sum_{i=1}^{k} r_{i} \leq 2, \sup _{y, x}\left|y f_{\varepsilon \mid X}^{\prime}(y \mid x)\right|<\infty, \sup _{y, x}\left|y \dot{f}_{\varepsilon \mid X}^{\prime}(y \mid x)\right|<\infty$ and $\sup _{y, x}\left|y^{2} f_{\varepsilon \mid X}^{\prime \prime}(y \mid x)\right|<\infty$.

(a7) For all $\eta>0$, there exists $\epsilon(\eta)>0$ such that $\inf _{\left\|\vartheta-\vartheta_{0}\right\|>\eta}\|G(\vartheta)\| \geq \epsilon(\eta)>0$. Moreover, the matrix $\Gamma$ defined in Theorem 2.1 is of full rank.

For the results of section 3, we will need assumptions (a1), (a2) and the following conditions. Let $\|\cdot\|$ denote some vector or matrix norm, depending on the object.

(A1) All partial derivatives of $F_{X}$ up to order $2 d+1$ exist on the interior of its compact support $R_{X}$, they are uniformly continuous and $\inf _{x \in R_{X}} f_{X}(x)>0$.

(A2) All partial derivatives of $m$ and $\sigma$ up to order $p+2$ exist on the interior of $R_{X}$, they are uniformly continuous and $\inf _{x \in R_{X}} \sigma(x)>0$.

(A3) $F_{\varepsilon}$ is twice continuously differentiable, $\sup _{y}\left|y f_{\varepsilon}(y)\right|<\infty, \sup _{y}\left|y^{2} f_{\varepsilon}^{\prime}(y)\right|<\infty$, and $E\left(\varepsilon^{6}\right)<\infty$.

(A4) $\sup _{y \in \mathbb{R}} E\left[\left\|\left.\nabla_{\vartheta} F_{\varepsilon(\vartheta) \mid X}(y \mid X)\right|_{\vartheta=\vartheta_{0}}\right\|\right]<\infty$

(A5) For the parameter estimator a linear expansion as in (3.2) is valid with $E\left[g_{\vartheta_{0}}(X, Y)\right]=$ $0, E\left[\left\|g_{\vartheta_{0}}(X, Y)\right\|^{2}\right]<\infty$.

(A6) Let $F_{Y \mid X}(\cdot \mid x)$ and $f_{Y \mid X}(\cdot \mid x)$ denote the conditional distribution and density function of $Y$, given $X=x$, respectively. We assume existence of some $\eta>0$ such that

$$
\sup _{\vartheta:\left\|\vartheta-\vartheta_{0}\right\| \leq \eta} \sup _{z \in \mathbb{R}} \int\left(\left|f_{Y \mid X}^{\prime}\left(V_{\vartheta}(z) \mid u\right)\right|\left\|\dot{V}_{\vartheta}(z)\right\|^{2}+f_{Y \mid X}\left(V_{\vartheta}(z) \mid u\right)\left\|\ddot{V}_{\vartheta}(z)\right\|\right) d F_{X}(x)<\infty .
$$

Here we use the notation $V_{\vartheta}=\Lambda_{\vartheta}^{-1}$ for the inverse of the transformation and $\dot{V}_{\vartheta}=$ $\nabla_{\vartheta} V_{\vartheta}$ and $\ddot{V}_{\vartheta}=\left(\frac{\partial^{2} V_{\vartheta}}{\partial \vartheta_{i} \vartheta_{j}}\right)_{i, j=1, \ldots, k}$ for the gradiant and Hessian matrix, respectively. Further we assume that $\sup _{y \in \mathbb{R}, x \in R_{X}}\left\|y \frac{\partial\left(f_{Y \mid X}\left(V_{\vartheta_{0}}(y) \mid x\right) \dot{V}_{\vartheta_{0}}(y)\right)}{\partial y}\right\|<\infty$. 
(A7) For some $\eta>0, E\left[\sup _{\vartheta:\left\|\vartheta-\vartheta_{0}\right\| \leq \eta}\left\|\ddot{\Lambda}_{\vartheta}(Y)\right\|\right]<\infty, E\left[\sup _{\vartheta:\left\|\vartheta-\vartheta_{0}\right\| \leq \eta}\left\|\dot{\Lambda}_{\vartheta}(Y)\right\|^{2}\right]<\infty$ and $E\left[\sup _{\vartheta:\left\|\vartheta-\vartheta_{0}\right\| \leq \eta}\left\|\ddot{\Lambda}_{\vartheta}(Y) \Lambda_{\vartheta}(Y)\right\|\right]<\infty$. Further,

$$
\begin{aligned}
& E\left[\sup _{\vartheta:\left\|\vartheta-\vartheta_{0}\right\| \leq \eta}\left\|\Lambda_{\vartheta}(Y) \dot{\Lambda}_{\vartheta}(Y)\right\| \mid X=x\right]<\infty \\
& E\left[\sup _{\vartheta:\left\|\vartheta-\vartheta_{0}\right\| \leq \eta}\left\|\dot{\Lambda}_{\vartheta}(Y)\right\| \mid X=x\right]<\infty
\end{aligned}
$$

for almost all $x \in R_{X}$.

(A8) Assumption (A2) holds with $m$ replaced by $E\left[\left.\frac{\partial \Lambda_{\vartheta}(Y)}{\partial \vartheta_{i}}\right|_{\vartheta=\vartheta_{0}} \mid X=\cdot\right]$ and $\sigma$ replaced by $E\left[\left.\Lambda_{\vartheta_{0}}(Y) \frac{\partial \Lambda_{\vartheta}(Y)}{\partial \vartheta_{i}}\right|_{\vartheta=\vartheta_{0}} \mid X=\cdot\right]$, for $i=1, \ldots, k$. Further, $E\left[\left\|\dot{\Lambda}_{\vartheta_{0}}(Y)\right\|^{3}\right]<\infty$ and $E\left[\left\|\Lambda_{\vartheta_{0}}(Y) \dot{\Lambda}_{\vartheta_{0}}(Y)\right\|^{3}\right]<\infty$.

Remarks on the assumptions. Assumptions (a1)-(a7) are needed to obtain the asymptotic result for the profile likelihood estimator $\hat{\vartheta}$ (Theorem 2.1). Here, (a1)-(a3) represent our possibilities to choose bandwidths and kernel functions. Assumptions (a4)(a7) are regularity conditions on the model and are analogous to assumptions A.1-A.8 by Linton et al. (2008) with some changes due to heteroscedasticity of our model and application of local polynomial estimators. For the asymptotic result on the estimated independence empirical process (Theorem 3.1), we reuse assumptions (a1) and (a2) concerning the choice of bandwidths and kernel for the estimation of the regression and variance function. However, instead of (a3)-(a7) we formulate assumption (A5) about the linear expansion of the estimator $\hat{\vartheta}$. Thus the application of Theorem 3.1 for different estimators $\hat{\vartheta}$ apart from the profile likelihood estimator is possible. Assumptions (a1), (a2), (A1)-(A3) are typically needed for weak convergence of empirical residual processes, compare to assumptions (C1)-(C5) by Neumeyer and Van Keilegom (2010) in a model without transformation. Additionally, (A4) is needed for some Taylor expansion of the, now $\vartheta$-dependent, error distribution with respect to $\vartheta$. Assumptions (A6)-(A8) are needed to prove asymptotic expansions of the empirical process, using Taylor expansions with respect to $\vartheta$. In particular they allow interchanging derivatives with integrals that appear in several terms. For specific classes of transformations in applications, those technical assumptions can be reformulated or replaced by simpler conditions. For example, assumption (A7) for the Yeo-Johnson family used in the simulations can be deduced from (conditional) moment assumptions on the observations $Y$. 


\section{B Proof of the main results}

\section{B.1 Proof of Theorem 3.1}

Let $\hat{F}_{X, \varepsilon}$ denote the joint empirical distribution function of $\left(X_{i}, \varepsilon_{i}\right), i=1, \ldots, n$, under $H_{0}$. Let further

$$
R_{n}(x, y)=E\left[I\{X \leq x\} I\left\{\Lambda_{\hat{\vartheta}}(Y) \leq y \hat{\sigma}(X)+\hat{m}(X)\right\} \mid \mathcal{Y}_{n}\right]-E[I\{X \leq x\} I\{\varepsilon \leq y\}]
$$

where $\mathcal{Y}_{n}=\left\{\left(X_{i}, Y_{i}\right) \mid i=1, \ldots, n\right\}$. Then we have the following Lemma.

Lemma B.1 Under the assumptions of Theorem 3.1,

$$
\hat{F}_{X, \hat{\varepsilon}}(x, y)=\hat{F}_{X, \varepsilon}(x, y)+R_{n}(x, y)+o_{P}\left(\frac{1}{\sqrt{n}}\right)
$$

uniformly with respect to $x \in R_{X}, y \in \mathbb{R}$.

Proof of Lemma B.1 Before starting the proof, we introduce some notations. For $k=\left(k_{1}, \ldots, k_{d}\right) \in \mathbb{N}_{0}^{d}$, let $k .=\sum_{j=1}^{d} k_{j}, D^{k}=\partial^{k \cdot} / \partial x_{1}^{k_{1}} \ldots \partial x_{d}^{k_{d}}$, and

$$
\|f\|_{d+\alpha}=\max _{k . \leq d} \sup _{x \in R_{X}}\left|D^{k} f(x)\right|+\max _{k .=d} \sup _{x, x^{\prime} \in R_{X}} \frac{\left|D^{k} f(x)-D^{k} f\left(x^{\prime}\right)\right|}{\left\|x-x^{\prime}\right\|^{\alpha}},
$$

where $\|\cdot\|$ is the Euclidean norm in $\mathbb{R}^{d}$. Further, let $\mathcal{G}_{1}=C_{1}^{d+\alpha}\left(R_{X}\right)$ be the class of $d$ times differentiable functions $f$ defined on $R_{X}$ such that $\|f\|_{d+\alpha} \leq 1$, and $\mathcal{G}_{2}=\tilde{C}_{2}^{d+\alpha}\left(R_{X}\right)$ be the class of $d$ times differentiable functions $f$ defined on $R_{X}$ such that $\|f\|_{d+\alpha} \leq 2$ and $\inf _{x \in R_{X}} f(x) \geq 1 / 2$. Finally, let

$$
\varphi_{\vartheta, g_{1}, g_{2}, y}(X, Y)=I\left\{\frac{\Lambda_{\vartheta}(Y)-m(X)}{\sigma(X)} \leq y g_{2}(X)+g_{1}(X)\right\}-I\left\{\frac{\Lambda_{\vartheta_{0}}(Y)-m(X)}{\sigma(X)} \leq y\right\} .
$$

With these notations, we have

$$
\sqrt{n}\left(\hat{F}_{X, \hat{\varepsilon}}(x, y)-\hat{F}_{X, \varepsilon}(x, y)-R_{n}(x, y)\right)=G_{n}(x, \hat{\vartheta},(\hat{m}-m) / \sigma, \hat{\sigma} / \sigma, y)
$$

where the empirical process

$G_{n}\left(x, \vartheta, g_{1}, g_{2}, y\right)=\frac{1}{\sqrt{n}} \sum_{i=1}^{n}\left(I\left\{X_{i} \leq x\right\} \varphi_{\vartheta, g_{1}, g_{2}, y}\left(X_{i}, Y_{i}\right)-E\left[I\{X \leq x\} \varphi_{\vartheta, g_{1}, g_{2}, y}(X, Y)\right]\right)$ (indexed in $x \in R_{X}, \vartheta \in \Theta, g_{1} \in \mathcal{G}_{1}, g_{2} \in \mathcal{G}_{2}, y \in \mathbb{R}$ ) converges weakly to a Gaussian process. This follows from Proposition S2.1 in the supplementary document, the Donsker property of $\left\{I\{X \leq x\} \mid x \in R_{X}\right\}$ and because products of uniformly bounded Donsker 
classes are Donsker (see Example 2.10 .8 in van der Vaart \& Wellner, 1996, p. 192). Thus $G_{n}$ is asymptotically stochastically equicontinuous with respect to

$$
\begin{aligned}
& \rho\left(\left(x, \vartheta, g_{1}, g_{2}, y\right),\left(x^{\prime}, \vartheta^{\prime}, g_{1}^{\prime}, g_{2}^{\prime}, y^{\prime}\right)\right) \\
= & \left(\operatorname{Var}\left(I\{X \leq x\} \varphi_{\vartheta, g_{1}, g_{2}, y}(X, Y)-I\left\{X \leq x^{\prime}\right\} \varphi_{\vartheta^{\prime}, g_{1}^{\prime}, g_{2}^{\prime}, y^{\prime}}(X, Y)\right)\right)^{1 / 2}
\end{aligned}
$$

(see van der Vaart, 1998, p. 262/263). We have

$$
\rho\left((x, \hat{\vartheta},(\hat{m}-m) / \sigma, \hat{\sigma} / \sigma, y),\left(x, \vartheta_{0}, 0,1, y\right)\right)=o_{P}\left(\delta_{n}\right)
$$

where $\delta_{n} \searrow 0$ by Proposition S2.3 in the supplementary document. Thus and because $\varphi_{\vartheta_{0}, 0,1, y} \equiv 0$ it follows that

$$
\begin{aligned}
& P\left(\sup _{x, y}\left|\sqrt{n}\left(\hat{F}_{X, \hat{\varepsilon}}(x, y)-\hat{F}_{X, \varepsilon}(x, y)-R_{n}(x, y)\right)\right|>\eta\right) \\
\leq & P\left(\sup _{\rho\left(\left(x, \vartheta, g_{1}, g_{2}, y\right),\left(x^{\prime}, \vartheta^{\prime}, g_{1}^{\prime}, g_{2}^{\prime}, y^{\prime}\right)\right) \leq \delta_{n}}\left|G_{n}\left(x, \vartheta, g_{1}, g_{2}, y\right)-G_{n}\left(x^{\prime}, \vartheta^{\prime}, g_{1}^{\prime}, g_{2}^{\prime}, y^{\prime}\right)\right|>\eta\right)
\end{aligned}
$$

which converges to zero for $n \rightarrow \infty$, for all $\eta>0$. From this the assertion of Lemma B.1 follows.

To finish the proof of Theorem 3.1 we decompose $R_{n}=A_{n}+B_{n}+C_{n}$, where

$$
\begin{aligned}
A_{n}(x, y)= & E\left[I\{X \leq x\} I\left\{\Lambda_{\hat{\vartheta}}(Y) \leq y \hat{\sigma}(X)+\hat{m}(X)\right\} \mid \mathcal{Y}_{n}\right] \\
& -E\left[I\{X \leq x\} I\left\{\Lambda_{\vartheta_{0}}(Y) \leq y \hat{\sigma}(X)+\hat{m}(X)\right\} \mid \mathcal{Y}_{n}\right] \\
B_{n}(x, y)= & E\left[I\{X \leq x\} I\left\{\Lambda_{\vartheta_{0}}(Y) \leq y \hat{\sigma}_{\hat{\vartheta}}(X)+\hat{m}_{\hat{\vartheta}}(X)\right\} \mid \mathcal{Y}_{n}\right] \\
& -E\left[I\{X \leq x\} I\left\{\Lambda_{\vartheta_{0}}(Y) \leq y \hat{\sigma}_{\vartheta_{0}}(X)+\hat{m}_{\vartheta_{0}}(X)\right\} \mid \mathcal{Y}_{n}\right] \\
C_{n}(x, y)= & E\left[I\{X \leq x\} I\left\{\Lambda_{\vartheta_{0}}(Y) \leq y \hat{\sigma}_{\vartheta_{0}}(X)+\hat{m}_{\vartheta_{0}}(X)\right\} \mid \mathcal{Y}_{n}\right] \\
& -E\left[I\{X \leq x\} I\left\{\Lambda_{\vartheta_{0}}(Y) \leq y \sigma_{\vartheta_{0}}(X)+m_{\vartheta_{0}}(X)\right\}\right] .
\end{aligned}
$$

For the ease of notation in the following let the parameter $\vartheta$ be one-dimensional. We use the same notations as in assumption (A6). Then we have

$$
A_{n}(x, y)=\int\left(F_{Y \mid X}\left(V_{\hat{\vartheta}}(y \hat{\sigma}(u)+\hat{m}(u)) \mid u\right)-F_{Y \mid X}\left(V_{\vartheta_{0}}(y \hat{\sigma}(u)+\hat{m}(u)) \mid u\right)\right) I\{u \leq x\} d F_{X}(u) .
$$

For the moment fix $u$ and $z=y \hat{\sigma}(u)+\hat{m}(u)$ and consider a second order Taylor expansion of the map $\vartheta \mapsto \psi(\vartheta)=F_{Y \mid X}\left(V_{\vartheta}(z) \mid u\right)$, i. e.

$$
\begin{aligned}
\psi(\hat{\vartheta})-\psi\left(\vartheta_{0}\right)= & f_{Y \mid X}\left(V_{\vartheta_{0}}(z) \mid u\right) \dot{V}_{\vartheta_{0}}(z)\left(\hat{\vartheta}-\vartheta_{0}\right) \\
& +\frac{1}{2}\left(f_{Y \mid X}^{\prime}\left(V_{\vartheta^{*}}(z) \mid u\right)\left(\dot{V}_{\vartheta^{*}}(z)\right)^{2}+f_{Y \mid X}\left(V_{\vartheta^{*}}(z) \mid u\right) \ddot{V}_{\vartheta^{*}}(z)\right)\left(\hat{\vartheta}-\vartheta_{0}\right)^{2} .
\end{aligned}
$$


The value $\vartheta^{*}$ may depend on $u$ and $z$, but lies between $\hat{\vartheta}$ and $\vartheta_{0}$. Because for each $\eta>0$, $\left|\hat{\vartheta}-\vartheta_{0}\right| \leq \eta$ with probability converging to one, for the proof we may assume $\left|\vartheta^{*}-\vartheta_{0}\right| \leq \eta$ with $\eta$ from assumption (A6). A Taylor expansion of $\psi$ motivates the definition of

$$
\tilde{A}_{n}(x, y)=\int f_{Y \mid X}\left(V_{\vartheta_{0}}(y \hat{\sigma}(u)+\hat{m}(u)) \mid u\right) \dot{V}_{\vartheta_{0}}(y \hat{\sigma}(u)+\hat{m}(u)) I\{u \leq x\} d F_{X}(u)\left(\hat{\vartheta}-\vartheta_{0}\right)
$$

and yields that

$$
\begin{aligned}
& \sup _{x, y}\left|A_{n}(x, y)-\tilde{A}_{n}(x, y)\right| \\
\leq & \left(\hat{\vartheta}-\vartheta_{0}\right)^{2} \frac{1}{2} \sup _{\vartheta:\left|\vartheta-\vartheta_{0}\right| \leq \eta} \sup _{z \in \mathbb{R}} \int\left(\mid\left(f_{Y \mid X}^{\prime}\left(V_{\vartheta}(z) \mid u\right)\left|\left(\dot{V}_{\vartheta}(z)\right)^{2}+f_{Y \mid X}\left(V_{\vartheta}(z) \mid u\right)\right| \ddot{V}_{\vartheta}(z) \mid\right) d F_{X}(x)\right. \\
= & o_{P}\left(\frac{1}{\sqrt{n}}\right)
\end{aligned}
$$

by assumption (A6). Denote by $\bar{A}_{n}$ the same term as $\tilde{A}_{n}$, but with the estimators $\hat{\sigma}$ and $\hat{m}$ replaced by the true functions $\sigma$ and $m$, respectively. Note that from the proof of Proposition S2.2 in the supplementary document uniform convergence of $|\hat{\sigma}-\sigma|$ and $|\hat{m}-m|$ to zero in probability follows and thus by the mean value theorem, the last part of assumption (A6), and $\hat{\vartheta}-\vartheta_{0}=O_{P}\left(n^{-1 / 2}\right)$ we obtain $\sup _{x, y}\left|\tilde{A}_{n}(x, y)-\bar{A}_{n}(x, y)\right|=$ $o_{P}\left(n^{-1 / 2}\right)$. Altogether for $A_{n}$ we have uniformly with respect to $x \in R_{X}, y \in \mathbb{R}$,

$$
\begin{aligned}
A_{n}(x, y)= & \int f_{Y \mid X}\left(V_{\vartheta_{0}}(y \sigma(u)+m(u)) \mid u\right) \dot{V}_{\vartheta_{0}}(y \sigma(u)+m(u)) I\{u \leq x\} d F_{X}(u)\left(\hat{\vartheta}-\vartheta_{0}\right) \\
& +o_{P}\left(\frac{1}{\sqrt{n}}\right) .
\end{aligned}
$$

For $C_{n}$ we obtain the following expansion uniformly with respect to $x, y$,

$$
\begin{aligned}
C_{n}(x, y)= & E\left[I\{X \leq x\} I\left\{\varepsilon \leq y \frac{\hat{\sigma}_{\vartheta_{0}}(X)}{\sigma(X)}+\frac{\hat{m}_{\vartheta_{0}}(X)-m(X)}{\sigma(X)}\right\} \mid \mathcal{Y}_{n}\right] \\
& -E[I\{X \leq x\} I\{\varepsilon \leq y\}] \\
= & \int\left(F_{\varepsilon}\left(y \frac{\hat{\sigma}_{\vartheta_{0}}(u)}{\sigma(u)}+\frac{\hat{m}_{\vartheta_{0}}(u)-m(u)}{\sigma(u)}\right)-F_{\varepsilon}(y)\right) I\{u \leq x\} d F_{X}(u) \\
= & f_{\varepsilon}(y)\left(y \int \frac{\hat{\sigma}_{\vartheta_{0}}(u)-\sigma(u)}{\sigma(u)} I\{u \leq x\} d F_{X}(u)\right. \\
& \left.+\int \frac{\hat{m}_{\vartheta_{0}}(u)-m(u)}{\sigma(u)} I\{u \leq x\} d F_{X}(u)\right)+o_{P}\left(\frac{1}{\sqrt{n}}\right) \\
= & f_{\varepsilon}(y) \frac{1}{n} \sum_{i=1}^{n}\left(\varepsilon_{i}+\frac{y}{2}\left(\varepsilon_{i}^{2}-1\right)\right) \int \frac{1}{h} K^{*}\left(\frac{u-X_{i}}{h}\right) I\{u \leq x\} d u+o_{P}\left(\frac{1}{\sqrt{n}}\right) .
\end{aligned}
$$


The second but last equality follows by Taylor's expansion, assumption (A3) and the fact that $\int\left(\hat{m}_{\vartheta_{0}}-m\right)^{2} / \sigma^{2} d F_{X}=o_{P}\left(n^{-1 / 2}\right), \int\left(\hat{\sigma}_{\vartheta_{0}}-\sigma\right)^{2} / \sigma^{2} d F_{X}=o_{P}\left(n^{-1 / 2}\right)$, see the proof of Theorem 2.1 in Neumeyer and Van Keilegom (2010). The last equality follows from (S1.1) and (S1.2) in the supplementary document, a combination of the proof of Lemma A.2 in Neumeyer and Van Keilegom (2010), and the proof of Proposition 2 (p. 537) in Neumeyer and Van Keilegom (2009).

Now let either $Z_{i}=\varepsilon_{i}$ or $Z_{i}=\varepsilon_{i}^{2}-1$. Then exactly as in the last part of the proof of Lemma B.1 in the supporting information to Birke and Neumeyer (2013) we have

$$
\sup _{x \in R_{X}}\left|\frac{1}{n} \sum_{i=1}^{n} Z_{i}\left(\int \frac{1}{h^{d}} K^{*}\left(\frac{u-X_{i}}{h}\right) I\{u \leq x\} d u-I\left\{X_{i} \leq x\right\}\right)\right|=o_{P}\left(\frac{1}{\sqrt{n}}\right) .
$$

Altogether for $C_{n}$ we have uniformly with respect to $x \in R_{X}, y \in \mathbb{R}$,

$$
C_{n}(x, y)=f_{\varepsilon}(y) \frac{1}{n} \sum_{i=1}^{n}\left(\varepsilon_{i}+\frac{y}{2}\left(\varepsilon_{i}^{2}-1\right)\right) I\left\{X_{i} \leq x\right\}+o_{P}\left(\frac{1}{\sqrt{n}}\right) .
$$

With $B_{n}$ we proceed similarly to obtain

$$
\begin{aligned}
B_{n}(x, y)= & f_{\varepsilon}(y)\left(y \int \frac{\hat{\sigma}_{\hat{\vartheta}}(u)-\hat{\sigma}_{\vartheta_{0}}(u)}{\sigma(u)} I\{u \leq x\} d F_{X}(u)\right. \\
& \left.+\int \frac{\hat{m}_{\hat{\vartheta}}(u)-\hat{m}_{\vartheta_{0}}(u)}{\sigma(u)} I\{u \leq x\} d F_{X}(u)\right)+o_{P}\left(\frac{1}{\sqrt{n}}\right)
\end{aligned}
$$

by assumption (A3) and the fact that $\sup _{x}\left|\hat{m}_{\hat{\vartheta}}(x)-\hat{m}_{\vartheta_{0}}(x)\right|=O_{P}\left(n^{-1 / 2}\right), \sup _{x} \mid \hat{\sigma}_{\hat{\vartheta}}(x)-$ $\hat{\sigma}_{\vartheta_{0}}(x) \mid=O_{P}\left(n^{-1 / 2}\right)$ (see the proof of Proposition S2.2). Now note that

$$
\begin{aligned}
\hat{m}_{\hat{\vartheta}}(u)-\hat{m}_{\vartheta_{0}}(u) & =\frac{1}{n h^{d}} \sum_{i=1}^{n} W_{u, n}\left(\frac{u-X_{i}}{h}\right)\left(\Lambda_{\hat{\vartheta}}\left(Y_{i}\right)-\Lambda_{\vartheta_{0}}\left(Y_{i}\right)\right) \\
& =\frac{1}{n h^{d}} \sum_{i=1}^{n} W_{u, n}\left(\frac{u-X_{i}}{h}\right) \dot{\Lambda}_{\vartheta_{0}}\left(Y_{i}\right)\left(\hat{\vartheta}-\vartheta_{0}\right)+r_{n}(u),
\end{aligned}
$$

where

$$
\begin{aligned}
& \int \frac{r_{n}(u)}{\sigma(u)} I\{u \leq x\} d F_{X}(u) \\
& \leq \frac{1}{2}\left(\hat{\vartheta}-\vartheta_{0}\right)^{2} \int \frac{1}{n h^{d}} \sum_{i=1}^{n}\left|W_{u, n}\left(\frac{u-X_{i}}{h}\right)\right| \sup _{\vartheta:\left|\vartheta-\vartheta_{0}\right| \leq \eta}\left|\ddot{\Lambda}_{\vartheta}\left(Y_{i}\right)\right| \frac{I\{u \leq x\}}{\sigma(u)} d F_{X}(u) \\
& =o_{P}\left(n^{-1 / 2}\right)
\end{aligned}
$$


by assumptions (A5) and (A7). Proceeding similarly to the expansion of $C_{n}$ we thus obtain

$$
\begin{aligned}
& \int \frac{\hat{m}_{\hat{\vartheta}}(u)-\hat{m}_{\vartheta_{0}}(u)}{\sigma(u)} I\{u \leq x\} d F_{X}(u) \\
= & \left(\hat{\vartheta}-\vartheta_{0}\right) \frac{1}{n} \sum_{i=1}^{n} \dot{\Lambda}_{\vartheta_{0}}\left(Y_{i}\right) \int \frac{1}{h^{d}} K^{*}\left(\frac{u-X_{i}}{h}\right) \frac{I\{u \leq x\}}{\sigma(u)} d x+o_{P}\left(\frac{1}{\sqrt{n}}\right) \\
= & \left(\hat{\vartheta}-\vartheta_{0}\right) E\left[\dot{\Lambda}_{\vartheta_{0}}(Y) \frac{I\{X \leq x\}}{\sigma(X)}\right]+o_{P}\left(\frac{1}{\sqrt{n}}\right) .
\end{aligned}
$$

Similarly for the variance we have $\hat{\sigma}_{\hat{\vartheta}}-\hat{\sigma}_{\vartheta_{0}}=\left(\hat{\sigma}_{\hat{\vartheta}}^{2}-\hat{\sigma}_{\vartheta_{0}}^{2}\right) /\left(\hat{\sigma}_{\hat{\vartheta}}+\hat{\sigma}_{\vartheta_{0}}\right)$ which yields (compare to $($ B.1))

$$
\begin{aligned}
& \int \frac{\hat{\sigma}_{\hat{\vartheta}}(u)-\hat{\sigma}_{\vartheta_{0}}(u)}{\sigma(u)} I\{u \leq x\} d F_{X}(u) \\
= & \frac{1}{2} \int \frac{1}{\sigma^{2}(u)} \frac{1}{n h^{d}} \sum_{i=1}^{n} W_{u, n}\left(\frac{u-X_{i}}{h}\right)\left(\left(\Lambda_{\hat{\vartheta}}\left(Y_{i}\right)\right)^{2}-\left(\Lambda_{\vartheta_{0}}\left(Y_{i}\right)\right)^{2}\right) I\{u \leq x\} d F_{X}(u) \\
& +\frac{1}{2} \int \frac{1}{\sigma^{2}(u)}\left(\hat{m}_{\vartheta_{0}}(u)-\hat{m}_{\hat{\vartheta}}(u)\right)\left(\hat{m}_{\vartheta_{0}}(u)+\hat{m}_{\hat{\vartheta}}(u)\right) I\{u \leq x\} d F_{X}(u)+o_{P}\left(\frac{1}{\sqrt{n}}\right) \\
= & \left(\hat{\vartheta}-\vartheta_{0}\right)\left(\left.\frac{1}{2 n} \sum_{i=1}^{n} \frac{\partial\left(\Lambda_{\vartheta}\left(Y_{i}\right)\right)^{2}}{\partial \vartheta}\right|_{\vartheta=\vartheta_{0}} \int \frac{1}{h^{d}} K^{*}\left(\frac{u-X_{i}}{h}\right) \frac{I\{u \leq x\}}{\sigma^{2}(u)} d u\right. \\
& \left.-\frac{1}{2 n} \sum_{i=1}^{n} \dot{\Lambda}_{\vartheta_{0}}\left(Y_{i}\right) \int \frac{1}{h^{d}} K^{*}\left(\frac{u-X_{i}}{h}\right) \frac{I\{u \leq x\}}{\sigma^{2}(u)} 2 m(u) d u\right)+o_{P}\left(\frac{1}{\sqrt{n}}\right) \\
= & \left(\hat{\vartheta}-\vartheta_{0}\right) \frac{1}{n} \sum_{i=1}^{n}\left(\dot{\Lambda}_{\vartheta_{0}}\left(Y_{i}\right) \Lambda_{\vartheta_{0}}\left(Y_{i}\right)-\dot{\Lambda}_{\vartheta_{0}}\left(Y_{i}\right) m\left(X_{i}\right)\right) \frac{I\left\{X_{i} \leq x\right\}}{\sigma^{2}\left(X_{i}\right)}+o_{P}\left(\frac{1}{\sqrt{n}}\right) \\
= & \left(\hat{\vartheta}-\vartheta_{0}\right) E\left[\left(\dot{\Lambda}_{\vartheta_{0}}(Y) \Lambda_{\vartheta_{0}}(Y)-\dot{\Lambda}_{\vartheta_{0}}(Y) m(X)\right) \frac{I\{X \leq x\}}{\sigma^{2}(X)}\right]+o_{P}\left(\frac{1}{\sqrt{n}}\right) .
\end{aligned}
$$

Those expansions yield uniformly with respect to $x$ and $y$,

$$
\begin{aligned}
B_{n}(x, y)= & \left(\hat{\vartheta}-\vartheta_{0}\right) f_{\varepsilon}(y) E\left[\dot{\Lambda}_{\vartheta_{0}}(Y)\left(\sigma(X)+y \Lambda_{\vartheta_{0}}(Y)-y m(X)\right) \frac{I\{X \leq x\}}{\sigma^{2}(X)}\right] \\
& +o_{P}\left(\frac{1}{\sqrt{n}}\right) .
\end{aligned}
$$

The expansions derived for $A_{n}, B_{n}$ and $C_{n}$ now yield

$$
\begin{aligned}
R_{n}(x, y)= & \left(\hat{\vartheta}-\vartheta_{0}\right) H_{\vartheta_{0}}(x, y)+f_{\varepsilon}(y) \frac{1}{n} \sum_{i=1}^{n}\left(\varepsilon_{i}+\frac{y}{2}\left(\varepsilon_{i}^{2}-1\right)\right) I\left\{X_{i} \leq x\right\} \\
& +o_{P}\left(\frac{1}{\sqrt{n}}\right)
\end{aligned}
$$


with

$$
\begin{aligned}
H_{\vartheta_{0}}(x, y)= & f_{\varepsilon}(y) E\left[\dot{\Lambda}_{\vartheta_{0}}(Y)\left(\sigma(X)+y \Lambda_{\vartheta_{0}}(Y)-y m(X)\right) \frac{I\{X \leq x\}}{\sigma^{2}(X)}\right] \\
& +\int f_{Y \mid X}\left(V_{\vartheta_{0}}(y \sigma(u)+m(u)) \mid u\right) \dot{V}_{\vartheta_{0}}(y \sigma(u)+m(u)) I\{u \leq x\} d F_{X}(u) \\
= & E\left[\left.\frac{\partial}{\partial \vartheta} F_{\varepsilon(\vartheta) \mid X}(y \mid X)\right|_{\vartheta=\vartheta_{0}} I\{X \leq x\}\right] .
\end{aligned}
$$

The last equality follows by some tedious but straightforward calculations. Now the assertion of Theorem 3.1 follows by Lemma B.1, (B.2) and assumption (A5).

\section{B.2 Proof of Corollary 3.3}

From expansion (3.4) we have

$$
S_{n}(x, y)=G_{n}\left(x, y, f_{\varepsilon}(y), y f_{\varepsilon}(y), h_{\vartheta_{0}}(x, y)\right)+o_{P}(1)
$$

uniformly, where

$$
h_{\vartheta_{0}}(x, y)=E\left[\left.\nabla_{\vartheta} F_{\varepsilon(\vartheta) \mid X}(y \mid X)\right|_{\vartheta=\vartheta_{0}}\left(I\{X \leq x\}-F_{X}(x)\right)\right]
$$

and where the process

$$
\begin{aligned}
& G_{n}\left(x, y, z_{1}, z_{2}, z_{3}\right) \\
= & \frac{1}{\sqrt{n}} \sum_{i=1}^{n}\left(\left(I\left\{X_{i} \leq x\right\}-F_{X}(x)\right)\left(I\left\{\varepsilon_{i} \leq y\right\}-F_{\varepsilon}(y)+z_{1} \varepsilon_{i}+\frac{z_{2}}{2}\left(\varepsilon_{i}^{2}-1\right)\right)\right. \\
& \left.+z_{3} g_{\vartheta_{0}}\left(X_{i}, Y_{i}\right)\right)
\end{aligned}
$$

is indexed in $\mathcal{F}=\left\{\left(x, y, z_{1}, z_{2}, z_{3}\right) \mid x \in R_{X}, y \in \mathbb{R}, z_{1}, z_{2}, z_{3} \in[-K, K]\right\}$ for some $K$ such that $\sup _{y} f_{\varepsilon}(y) \leq K, \sup _{y}\left|y f_{\varepsilon}(y)\right| \leq K, \sup _{x, y}\left|h_{\vartheta_{0}}(x, y)\right| \leq K$ (see assumptions (A3) and (A4)). Weak convergence of $G_{n}$ follows similarly to the proof of Theorem 2 in Neumeyer and Van Keilegom (2009, p. 538). The key argument is that for the bracketing number $N_{[]}\left(\eta, \mathcal{F}, L_{2}(P)\right)$ an order $O\left(\eta^{-7}\right)$ can be derived from the $L_{2}(P)$-norm

$$
\begin{aligned}
& \left(E \left[\left(\left(I\left\{X_{i} \leq x\right\}-F_{X}(x)\right)\left(I\left\{\varepsilon_{i} \leq y\right\}-F_{\varepsilon}(y)+z_{1} \varepsilon_{i}+\frac{z_{2}}{2}\left(\varepsilon_{i}^{2}-1\right)\right)+z_{3} g_{\vartheta_{0}}\left(X_{i}, Y_{i}\right)\right.\right.\right. \\
& \left.\left.\left.-\left(I\left\{X_{i} \leq x^{\prime}\right\}-F_{X}\left(x^{\prime}\right)\right)\left(I\left\{\varepsilon_{i} \leq y^{\prime}\right\}-F_{\varepsilon}\left(y^{\prime}\right)+z_{1}^{\prime} \varepsilon_{i}+\frac{z_{2}^{\prime}}{2}\left(\varepsilon_{i}^{2}-1\right)\right)-z_{3}^{\prime} g_{\vartheta_{0}}\left(X_{i}, Y_{i}\right)\right)^{2}\right]\right)^{1 / 2} \\
& \leq C\left(\left|F_{X}(x)-F_{X}\left(x^{\prime}\right)\right|\left(1+K^{2}\left(1+\operatorname{Var}\left(\varepsilon^{2}\right)\right)\right)+\left|F_{\varepsilon}(y)-F_{\varepsilon}\left(y^{\prime}\right)\right|+\left(z_{1}-z_{1}^{\prime}\right)^{2}\right. \\
& \left.\quad+\left(z_{2}-z_{2}^{\prime}\right)^{2} \operatorname{Var}\left(\varepsilon^{2}\right)+\left(z_{3}-z_{3}^{\prime}\right)^{2} E\left[g_{\vartheta_{0}}^{2}(X, Y)\right]\right)^{1 / 2}
\end{aligned}
$$


for some constant $C$. Weak convergence of $S_{n}$ follows by consideration of the subclass of $\mathcal{F}$ defined by $z_{1}=f_{\varepsilon}(y), z_{2}=y f_{\varepsilon}(y), z_{3}=h_{\vartheta_{0}}(x, y)$.

\section{Acknowledgments}

The authors thank the co-editor, the associate editor and two reviewers for their helpful comments and suggestions and Oliver Linton for stimulating discussions regarding the profile likelihood method. The first author acknowledges financial support by the DFG (Research Unit FOR 1735 Structural Inference in Statistics: Adaptation and Effciency). The research of the second author was supported by Basic Science Research Program through the National Research Foundation of Korea (NRF) funded by the Ministry of Education (2014R1A1A2059875). The research of the third author was supported by the European Research Council under the European Community's Seventh Framework Programme (FP7/2007-2013) / ERC Grant agreement No. 203650, by IAP research network grant nr. P7/06 of the Belgian government (Belgian Science Policy), and by the contract 'Projet d'Actions de Recherche Concertées' (ARC) 11/16-039 of the 'Communauté française de Belgique', granted by the 'Académie universitaire Louvain'.

\section{References}

Akritas, M. G. and Van Keilegom, I. (2001). Non-parametric estimation of the residual distribution. Scand. J. Statist. 28, 549-567.

Azzalini, A. (2005). The skew-normal Distribution and Related Multivariate Families. Scand. J. Statist. 32, 159-188.

Bickel, P. J. and Doksum, K. A. (1981). An analysis of transformations revisited. J. Amer. Statist. Assoc. 76, 296-311.

Birke, M. and Neumeyer, N. (2013). Testing monotonicity of regression funtions - an empirical process approach. Scand. J. Statist. 40, 438-454.

Box, G. E. P. and Cox, D. R. (1964). An analysis of transformations. J. Roy. Statist. Soc. Ser. B 26, 211-252.

Carroll, R. J. and Ruppert, D. (1988). Transformation and Weighting in Regression. Monographs on Statistics and Applied Probability. Chapman \& Hall, New York.

Colling, B. and Van Keilegom, I. (2015). Goodness-of-fit tests in semiparametric transformation models. TEST, to appear. 
Dette, H., von Lieres und Wilkau, C. and Sperlich, S. (2005). A comparison of different nonparametric methods for inference on additive models. J. Nonparametr. Stat. 17, $57-81$.

Efromovich, S. (1999). Nonparametric Curve Estimation. Springer, New York.

Einmahl, J. H. J. and Van Keilegom, I. (2008). Specification tests in nonparametric regression. J. Econometrics 143, 88-102.

Fan, C. and Fine, J. P. (2013). Linear transformation model with parametric covariate transformations. J. Amer. Statist. Assoc. 108, 701-712.

Fan, J. and Gijbels, I. (1996). Local Polynomial Modelling and Its Applications. Chapman \& Hall, London.

Gijbels, I., Omelka, M. and Veraverbeke, N. (2015). Estimation of a copula when a covariate affects only marginal distributions. Scand. J. Statist., to appear.

González-Manteiga, W. and Crujeiras, R. M. (2013). An updated review of goodness-of-fit tests for regression models. TEST 22, 361-411.

Gu, J., Li, Q. and Yang, J-C. (2014). Multivariate local polynomial kernel estimators: leading bias and asymptotic distribution. Econometric Reviews, to appear.

Härdle, W. and Mammen, E. (1993). Comparing nonparametric versus parametric regression fits. Ann. Statist. 21, 1926-1947.

Hayfield, T. and Racine, J. S. (2008). Nonparametric Econometrics: The np Package. J. Stat. Softw. 27, URL http://www.jstatsoft.org/v27/i05/.

Heuchenne, C., Samb, R. and Van Keilegom, I. (2015). Estimating the residuals distribution in semiparametric transformation models. Electr. J. Statist., to appear.

Hlávka, Z., Hušková, M. and Meintanis, S. G. (2011). Test for independence in nonparametric heteroscedastic regression models. J. Multivariate Anal. 102, 816-827.

Horowitz, J. L. (1996). Semiparametric estimation of a regression model with an unknown transformation of the dependent variable. Econometrica 64, 103-137.

Horowitz, J. L. (2009). Semiparametric and nonparametric methods in econometrics. Springer Series in Statistics. Springer, New York.

Hušková, M. and Meintanis, S. G. (2010). Tests for the error distribution in nonparametric possibly heteroscedastic regression models. TEST 19, 92-112.

Khan, S., Shin, Y. and Tamer, E. (2011). Heteroskedastic Transformation Models with Covariate Dependent Censoring. Estimation on a semiparametric transformation model. J. Bus. Econ. Stat. 29, 40-48. 
Linton, O., Sperlich, S. and Van Keilegom, I. (2008). Estimation on a semiparametric transformation model. Ann. Statist. 36, 686-718.

Masry, E. (1996a). Multivariate local polynomial regression for time series: uniform strong consistency and rates. J. Time Ser. Anal. 17, 571-599.

Masry, E. (1996b). Multivariate regression estimation - local polynomial fitting for time series. Stochastic Process. Appl. 65, 81-101.

$\mathrm{Mu}, \mathrm{Y}$. and He, X. (2007). Power transformation toward a linear regression quantile. J. Amer. Statist. Assoc. 102, 269-279.

Neumeyer, N. (2009a). Testing independence in nonparametric regression. J. Multivariate Anal. 100, 1551-1566.

Neumeyer, N. (2009b). Smooth residual bootstrap for empirical processes of nonparametric regression residuals. Scand. J. Statist. 36, 204-228.

Neumeyer, N. and Sperlich, S. (2006). Comparison of separable components in different samples. Scand. J. Statist. 33, 477-501.

Neumeyer, N. and Van Keilegom, I. (2009). Change-point tests for the error distribution in nonparametric regression. Scand. J. Statist. 36, 518-541.

Neumeyer, N. and Van Keilegom, I. (2010). Estimating the error distribution in nonparametric multiple regression with applications to model testing. J. Multivariate Anal. 101, 1067-1078.

R Core Team (2015). R: A language and environment for statistical computing. R Foundation for Statistical Computing, Vienna, Austria. URL http://www.R-project.org/.

Ruppert, D., Sheather, S. J. and Wand, M. P. (1995). An effective bandwidth selector for local least squares regression. J. Amer. Statist. Assoc. 90, 1257-1270.

Severini, T. A. and Wong, W. H. (1992). Profile likelihood and conditinally parametric models. Ann. Statist., 20, 1768-1802.

Sheather, S. J. and Jones, M. C. (1991). A reliable data-based bandwidth selection method for kernel density estimation. J. Roy. Statist. Soc. Ser. B 53, 683-690.

Stute, W., González-Manteiga, W. and Presedo Quindimil, M. (1998). Bootstrap approximations in model checks for regression. J. Amer. Statist. Assoc. 93, 141-149.

Wand, M. (2015). KernSmooth: Functions for Kernel Smoothing Supporting Wand and Jones (1995). R package version 2.23-15. http://CRAN.R-project.org/package=KernSmooth

Van der Vaart, A. W. (1998). Asymptotic Statistics. Cambridge University Press. Cambridge. 
Van der Vaart, A. W. and Wellner, J. A. (1996). Weak Convergence and Empirical Processes. Springer-Verlag, New York.

Yeo, I-K. and Johnson, R. A. (2000). A new family of power transformations to improve normality or symmetry. Biometrika $\mathbf{8 7}, 954-959$.

Zhou, X-H., Lin, H. and Johnson, E. (2009). Non-parametric heteroscedastic transformation regression models for skewed data with an application to health care costs. $J$. Roy. Statist. Soc. Ser. B 70, 1029-1047.

Zhu, L., Fujikoshi, Y. and Naito, K. (2001). Heteroscedasticity checks for regression models. Sci. China Ser. A 44, 1236-1252. 